NISSUNA UMANA INVESTIGAZIONE SI PUO DIMANDARE VERA SCIENZIA S'ESSA NON PASSA PER LE MATEMATICHE DIMOSTRAZIONI LEONARDO DA VINCI

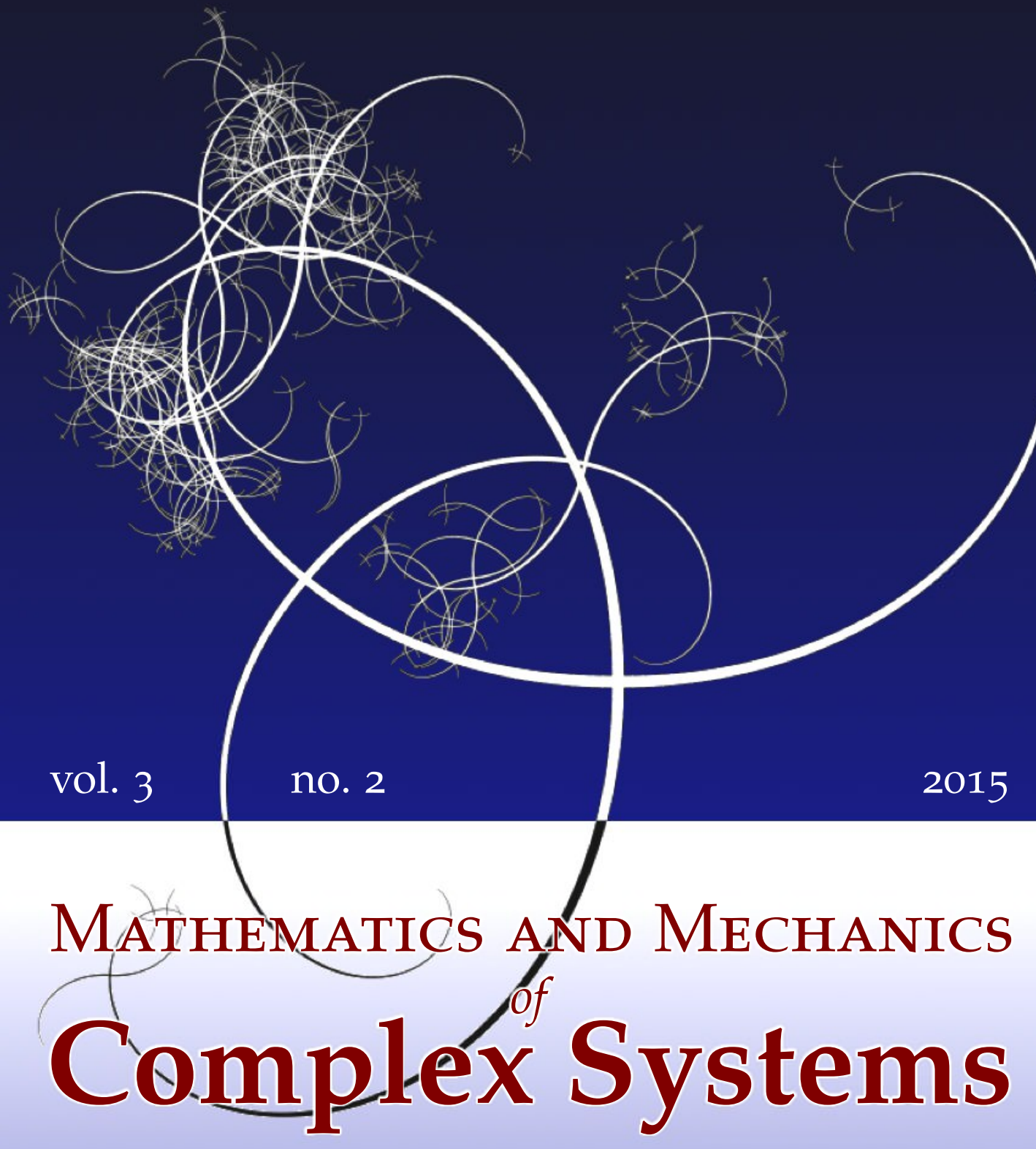

MARCO BENINI

RELATIVE CAUCHY EVOLUTION FOR THE VECTOR POTENTIAL ON GLOBALLY HYPERBOLIC SPACETIMES 


\title{
RELATIVE CAUCHY EVOLUTION FOR THE VECTOR POTENTIAL ON GLOBALLY HYPERBOLIC SPACETIMES
}

\author{
MARCO BENINI
}

\begin{abstract}
The dynamics of the electromagnetic vector potential is analyzed in full detail in view of the principle of general local covariance of Brunetti, Fredenhagen and Verch. Exploiting this result, the relative Cauchy evolution for the vector potential is introduced and its relation with the energy-momentum tensor is established, extending the well known results for Klein-Gordon and Dirac fields.
\end{abstract}

\section{Introduction}

The principle of general local covariance of Brunetti, Fredenhagen and Verch [Brunetti et al. 2003] provides a very satisfactory framework to deal with quantum field theory on curved spacetimes. The success of the axiomatic approach of general local covariance relies on its capability to establish how a quantum field theory is expected to behave on different spacetimes and in particular what kind of relation one should expect between the observables defined on two spacetimes when one of them is isometrically embedded into the other. An effective way being available to relate via embeddings quantum field theories on different curved spacetimes, the way is paved to tackle the question of the sensitivity of the model under small fluctuations of the background geometry. This is what the relative Cauchy evolution is meant for, namely to provide information about the modification induced on any observable by a small change in the metric of the background spacetime where the dynamics of the quantum field takes place.

The core idea of the relative Cauchy evolution can be traced back to the fact that a normally hyperbolic equation which rules the dynamics of a field on a globally hyperbolic spacetime admits a well-posed initial value problem; see for example [Bär et al. 2007, Section 3.2]. This means that all information about the field is determined by suitable initial data specified on a Cauchy surface, thus enabling us to sketch the behavior of the relative Cauchy evolution in terms of initial data only. One can consider a perturbation of the spacetime metric supported in a compact

\section{Communicated by Mauro Carfora.}

MSC2010: 81T20, 81T05, 81T13.

Keywords: quantum field theory on curved spacetimes, Maxwell equation, general local covariance, relative Cauchy evolution. 
region, say $K$, and a set of initial data on a Cauchy surface lying in the past of $K$. Propagating such initial data to a Cauchy surface in the future of $K$ both in the presence of the metric perturbation and without it, one is able to compare the two different outcomes of the dynamical evolution (one when the background geometry is perturbed and the other when this is not the case). This procedure allows one to quantify the effect induced on the dynamics of the field by suitable modifications of the background geometry.

The aim of the present paper is to study the relative Cauchy evolution for the gauge field theory of the electromagnetic vector potential. Given a globally hyperbolic spacetime, the dynamics of the field $A \in \Omega^{1}(M)$ is ruled by the nonhyperbolic equation $\delta \mathrm{d} A=0, \mathrm{~d}$ and $\delta$ being respectively the differential and the codifferential defined for forms over $M$. The gauge symmetry of the vector potential is specified by the equivalence relation

$$
A \sim A^{\prime} \Longleftrightarrow \exists \chi \in \mathrm{C}^{\infty}(M): A^{\prime}=A+\mathrm{d} \chi .
$$

Although the sketch of the relative Cauchy evolution presented above cannot be directly applied to the case of interest, the dynamics being nonhyperbolic, we can, following [Dimock 1992; Fewster and Pfenning 2003; Pfenning 2009; Dappiaggi 2011; Sanders et al. 2014], exploit the gauge symmetry to recover hyperbolicity in the gauge-fixed dynamics. This eventually leads us to the fulfillment of (almost all) requirements of the generally covariant locality principle. In particular, we are allowed to introduce the relative Cauchy evolution for the vector potential $A$ and analyze its properties, the main result consisting of the extension of a fact which is known to hold for Klein-Gordon and Dirac fields [Brunetti et al. 2003; Sanders 2010], namely the relation between the relative Cauchy evolution of a field and its quantized energy-momentum tensor.

Such a relation between the relative Cauchy evolution and the quantized energymomentum tensor is relevant when one is dealing with the semiclassical Einstein equation (see [Wald 1994, Section 4.6] for an introduction to this topic) in the presence of a quantized electromagnetic vector potential. As a matter of fact, in this case one is supposed to equal the Einstein tensor with the expectation value of the quantized energy-momentum tensor of electromagnetism in order to account for the back reaction effect on the spacetime metric induced by the presence of a quantized electromagnetic field, whose dynamics is in turn affected by the spacetime geometry. Fortunately, one can access the behavior of the quantized energymomentum tensor in relation to suitable changes of the background metric by means of the relative Cauchy evolution. Therefore an important step towards a consistent approach to the solution of the semiclassical Einstein equation in the presence of a quantized electromagnetic field consists of a detailed analysis of the relative Cauchy evolution for the electromagnetic field as well as of its relation 
with the quantized energy-momentum tensor. This fact motivates our interest in analyzing the relative Cauchy evolution of the vector potential.

The paper is organized in the following way: Section 2 is intended to provide the background information and notation which are needed in the rest of the paper. Some aspects of Lorentzian geometry are briefly discussed in Section 2A, focusing on global hyperbolicity in particular. A short collection of the most relevant properties of Green-hyperbolic linear differential operators follows in Section 2B. We recall the notion of a locally covariant quantum field theory and of its relative Cauchy evolution in Section 2C. Section 2 is completed with the description of a quantization procedure based on the Borchers-Uhlmann construction and recalling the notion of an algebraic state. In Section 3 we analyze the dynamics of the vector potential providing a convenient characterization of the space of solutions for the equation $\delta \mathrm{d} A=0$. This leads in Section 4 to the assignment of a suitable space of observables for the vector potential and its quantization. This section ends recalling the definition of a Hadamard state for the vector potential, together with references to the literature where positive results about the existence of such a state can be found. The core of the paper is Section 5, where the relative Cauchy evolution for the vector potential is computed and its relation with the quantized energymomentum tensor is established, thus extending a result which was already known to hold for Klein-Gordon [Brunetti et al. 2003] and Dirac fields [Sanders 2010].

\section{Preliminaries}

In this section we collect the background material and, at the same time, we introduce some notation needed later. First, we will briefly recall few notions about Lorentzian geometry focusing the attention on globally hyperbolic spacetimes, whose physical relevance is related to initial value problems for hyperbolic linear partial differential equations. As a matter of fact, globally hyperbolic spacetimes provide a sufficiently general setting for proving existence and uniqueness theorems for solutions of partial differential equations of hyperbolic type once proper initial data are given; see [Bär et al. 2007, Chapter 3]. This leads us to the second part of the present section, which is devoted to differential operators. We will focus the attention on the class of Green-hyperbolic operators, being characterized by the existence of retarded and advanced Green functions. We will take the chance to recollect from the literature few fundamental results, which will turn out to be useful throughout the rest of the paper. In the third part of this section, we first provide the framework for the relative Cauchy evolution, namely we introduce general local covariance following [Brunetti et al. 2003] and in particular the time slice axiom, and then we define it using a simple geometrical construction. Since in the end we are interested in the quantization of our model, we recall an 
algebraic procedure to assign canonical commutation relations, we define states from an algebraic perspective and we make contact with the usual Hilbert space representation of quantum field theory via the Gelfand-Naimark-Segal theorem.

2A. Lorentzian geometry. We recall here few basic notions of Lorentzian geometry, global hyperbolicity in particular, and we take the chance to introduce some notation. For a detailed analysis of these topics, the reader should refer to the literature; see, e.g.,[Beem et al. 1996; Bär et al. 2007; Waldmann 2012].

In the following all manifolds and functions between manifolds are considered to be smooth, unless otherwise stated. Sometimes we will also restrict ourselves to manifolds of finite type, namely manifolds admitting a finite good cover. This will enable us to fully recover full Poincaré duality; see [Bott and Tu 1982, Chapter 1, Section 5].

A Lorentzian manifold $(M, g, \mathfrak{o})$ is a $d$-dimensional, orientable, connected, secondcountable, Hausdorff manifold endowed with a Lorentzian metric $g$ and a choice of orientation $\mathfrak{o}$. We adopt the convention $-+\cdots+$ for the signature of $g$. This structure already enables us to distinguish among timelike, lightlike (all together causal) and spacelike tangent vectors $0 \neq v \in T_{x} M$ at a point $x \in M$ according to the negative, null or positive value of $g(v, v)$. Moreover, the choice of an orientation $\mathfrak{o}$, together with the metric $g$, uniquely identifies a volume form vol on $M$, which is used to integrate functions defined $M$.

In order to account for the dynamical evolution of a physical system, proper notions of future and past are required. This is achieved taking a time-orientable Lorentzian manifold and fixing a time-orientation specified by a timelike vector field $\mathfrak{t}$, which is used as a reference to distinguish between future- and past-directed causal tangent vectors $v$ according to the sign of $g(\mathfrak{t}, v)$ (future for negative values). The quadruple $(M, g, \mathfrak{o}, \mathfrak{t})$ defines a spacetime, where the notion of causal future/past of $O \subseteq M, J_{M}^{ \pm}(O)$, is available. $J_{M}^{ \pm}(O)$ is defined as the set of points in $M$ that can be reached via a future-/past-directed causal curve in $M$ emanating from $O$, namely a curve whose tangent vector field is everywhere causal and future/past-directed. If we take into account only timelike curves in the last definition, we obtain the chronological future/past of $O, I_{M}^{ \pm}(O)$. With the notion of causal future and past at hand, we can characterize subregions of $M$ which are compatible with the causal structure of $(M, g, \mathfrak{o}, \mathfrak{t})$, as well as maps between spacetimes preserving causal structures. Specifically, a region $S \subseteq M$ is called causally compatible provided that $J_{S}^{ \pm}(x)=J_{M}^{ \pm}(x) \cap S$ regardless of the choice of $x \in S .{ }^{1}$ Furthermore, a causal embedding $f$ between the spacetimes $\left(M_{1}, g_{1}, \mathfrak{o}_{1}, \mathfrak{t}_{1}\right)$ and $\left(M_{2}, g_{2}, \mathfrak{o}_{2}, \mathfrak{t}_{2}\right)$ is defined as an embedding $f: M_{1} \rightarrow M_{2}$ such that $f^{*} g_{2}=g_{1}$, which preserves

\footnotetext{
${ }^{1}$ Note that in the definition of $J_{S}^{ \pm}(x)$ only curves which never leave $S$ are taken into account.
} 
both the orientations and the time-orientations, whose image $f\left(M_{1}\right)$ is open and causally compatible as a subset of $M_{2}$.

In order to provide appropriate initial data for Cauchy problems a Cauchy surface is needed; see, for example, [Bär et al. 2007, Section 3.2]. This is a subset $\Sigma$ of a spacetime $(M, g, \mathfrak{o}, \mathfrak{t})$ which meets exactly once each inextensible future-directed timelike curve. Cauchy surfaces provide a definition of globally hyperbolic spacetimes. As a matter of fact, a spacetime $(M, g, \mathfrak{o}, \mathfrak{t})$ is called globally hyperbolic when it admits a Cauchy surface. In the following we will denote globally hyperbolic spacetimes with $M$, the metric $g$, the orientation $\mathfrak{o}$, and the time-orientation $\mathfrak{t}$ being understood.

For later purposes, we introduce here some nomenclature for spacetime subregions. Following [Bär 2013; Sanders 2013], we call a subset $S$ of a globally hyperbolic spacetime $M$ :

- spacelike-compact (sc) if it is closed and there exists $K \subseteq M$ compact such that $S \subseteq J_{M}(K)=J_{M}^{+}(K) \cup J_{M}^{-}(K)$;

- past-compact/future-compact (pc/fc) if $S \cap J_{M}^{\mp}(K)$ is compact for each compact subset $K$ of $M$.

If a region $S \subseteq M$ is both pc and fc, we call it timelike-compact (tc). If it is both $\mathrm{pc} / \mathrm{fc}$ and $\mathrm{sc}$, we say that it is strictly past-compact/strictly future-compact $(\mathrm{spc} / \mathrm{sfc})$.

2B. Green-hyperbolic differential operators. Following the definitions in [Bär and Ginoux 2012a; 2012b; Bär 2013], this is a class of linear differential operators admitting retarded and advanced Green functions on globally hyperbolic spacetimes. This class includes, of course, all wave operators, such as the d'Alembert operator $\square_{\nabla}=g^{\mu \nu} \nabla_{\mu} \nabla_{\nu}$ defined out of any connection $\nabla$ on a vector bundle, but from a physical perspective it has the advantage of encompassing other relevant cases, such as the Dirac and Proca equations.

Here we briefly review the definitions of retarded and advanced Green functions. At the same time we recall few fundamental results for the so-called causal propagator. For a detailed discussion, as well as proofs of the forthcoming statements, the reader may refer to the papers just cited, as well as [Bär et al. 2007]. A review, with some physically relevant examples, is available in [Benini et al. 2013].

Definition 2.1. Let $V$ and $W$ be vector bundles over a globally hyperbolic spacetime $M$ and consider a linear differential operator $P: \Gamma(V) \rightarrow \Gamma(W)$ defined between the corresponding spaces of sections. We call retarded/advanced Green operator for $P$ a linear map $G_{ \pm}: \Gamma_{\mathrm{c}}(W) \rightarrow \Gamma(V)$ such that the following conditions hold for each $\sigma \in \Gamma_{\mathrm{c}}(W)$ and $\tau \in \Gamma_{\mathrm{c}}(V)$ :

$$
P G_{ \pm} \sigma=\sigma, \quad G_{ \pm} P \tau=\tau, \quad \operatorname{supp}\left(G_{ \pm} \sigma\right) \subseteq J_{M}^{ \pm}(\operatorname{supp}(\sigma)) .
$$


If we endow $V$ with a nondegenerate inner product on the fibers, denoted by $\langle\cdot, \cdot\rangle_{V}: V \times V \rightarrow M \times \mathbb{R}$, and taking into account the volume form vol of $M$, we can introduce an inner product $(\cdot, \cdot)_{V}$ on the corresponding space of sections. This is defined according to

$$
(\sigma, \tau)_{V}=\int_{M}\langle\sigma, \tau\rangle_{V} \text { vol, }
$$

for each $\sigma, \tau \in \Gamma(V)$ with compact overlapping supports, namely such that $\operatorname{supp}(\sigma) \cap$ $\operatorname{supp}(\tau)$ is compact.

Given a linear differential operator $P: \Gamma(V) \rightarrow \Gamma(W)$ as in Definition 2.1 and assuming that both $V$ and $W$ are endowed with nondegenerate inner products, we can introduce the formal adjoint $P^{*}: \Gamma(W) \rightarrow \Gamma(V)$ of $P$ by setting

$$
\left(P^{*} \sigma, \tau\right)_{V}=(\sigma, P \tau)_{W},
$$

for each $\sigma \in \Gamma(W)$ and $\tau \in \Gamma(V)$ with compact overlapping support. We are now ready to define linear differential operators of Green-hyperbolic type.

Definition 2.2. Let $V, W$ be vector bundles over a globally hyperbolic spacetime $M$ endowed with nondegenerate inner products. A linear differential operator $P: \Gamma(V) \rightarrow \Gamma(W)$ is of Green-hyperbolic type if it admits retarded and advanced Green operators, together with its formal adjoint $P^{*}: \Gamma(W) \rightarrow \Gamma(V)$.

The fact that $P^{*}$ is the formal adjoint of $P$ entails a relation between the corresponding Green functions:

$$
\left(G_{ \pm}^{*} \sigma, \tau\right)_{W}=\left(\sigma, G_{\mp} \tau\right)_{V},
$$

for each $\sigma \in \Gamma_{\mathrm{c}}(V)$ and $\tau \in \Gamma_{\mathrm{c}}(W)$. As a consequence, retarded and advanced Green operators for both $P$ and $P^{*}$ are unique.

Moreover, Green functions for Green-hyperbolic operators admit unique continuous extensions to larger spaces of sections; see [Bär 2013, Section 3] and [Sanders 2013, Section 5]. With a slight abuse of notation, we denote with $G_{ \pm}$also the extended Green operators for $P$ :

$$
G_{+}: \Gamma_{\mathrm{pc}}(W) \rightarrow \Gamma(V), \quad G_{-}: \Gamma_{\mathrm{fc}}(W) \rightarrow \Gamma(V),
$$

the subscripts "pc" and "fc" referring to the supports of sections, which are pastcompact in the first case and future-compact in the second; see Section 2A. Extended Green operators share the same properties of the original ones, but in a broader sense: For each $\sigma \in \Gamma_{\mathrm{pc}}(W)$ and $\tau \in \Gamma_{\mathrm{pc}}(V)$, we have

$$
P G_{+} \sigma=\sigma, \quad G_{+} P \tau=\tau, \quad \operatorname{supp}\left(G_{+} \sigma\right) \subseteq J_{M}^{+}(\operatorname{supp}(\sigma)) .
$$


Replacing pc and + with $\mathrm{fc}$ and - , we get the properties of the extended Green function $G_{-}$. Similarly, the Green operators $G_{ \pm}^{*}$ for the formal adjoint $P^{*}$ admit unique extensions.

Introducing the causal propagator $G=G_{+}-G_{-}: \Gamma_{\mathrm{c}}(W) \rightarrow \Gamma(V)$ for a Greenhyperbolic operator $P: \Gamma(V) \rightarrow \Gamma(W)$ as the difference between the retarded and the advanced Green operators and taking into account the support properties of Green operators (see Definition 2.1), we realize that $G$ maps to $\Gamma_{\mathrm{sc}}(V)$, the space of sections with spacelike-compact support. We get the following exact sequence of vector spaces:

$$
0 \longrightarrow \Gamma_{\mathrm{c}}(V) \stackrel{P}{\longrightarrow} \Gamma_{\mathrm{c}}(W) \stackrel{G}{\longrightarrow} \Gamma_{\mathrm{sc}}(V) \stackrel{P}{\longrightarrow} \Gamma_{\mathrm{sc}}(W) \longrightarrow 0 .
$$

The proof of this fact easily follows from (2-6) and can be found, e.g., in [Bär et al. 2007, Section 3.4], except for surjectivity of $P: \Gamma_{\mathrm{sc}}(V) \rightarrow \Gamma_{\mathrm{sc}}(W)$, which is shown by the following argument; see also [Khavkine 2014b, Proposition 2.1]. Given $\tau \in \Gamma_{\mathrm{sc}}(W)$ and taking a partition of unity $\left\{\chi_{+}, \chi_{-}\right\}$on $M$ such that $\chi_{+}=1$ in a past-compact region, while $\chi_{-}=1$ in a future-compact one, we deduce that $\operatorname{supp}\left(\chi_{+} \tau\right)$ is strictly past-compact, while $\operatorname{supp}\left(\chi_{-} \tau\right)$ is strictly future-compact. Exploiting the extended Green operators, we are able to introduce a section

$$
\sigma=G_{+}\left(\chi_{+} \tau\right)+G_{-}\left(\chi_{-} \tau\right) \in \Gamma_{\mathrm{sc}}(V)
$$

such that $P \sigma=\tau$. This is a direct consequence of (2-6).

An exact sequence similar to (2-7) holds true for the causal propagator $G^{*}$ of the formal adjoint $P^{*}$ as well.

Extended Green operators provide also an extension of the causal propagator $G: \Gamma_{\mathrm{tc}}(W) \rightarrow \Gamma(V)$. Minor modifications to the proof of (2-7) give the following exact sequence:

$$
0 \longrightarrow \Gamma_{\mathrm{tc}}(V) \stackrel{P}{\longrightarrow} \Gamma_{\mathrm{tc}}(W) \stackrel{G}{\longrightarrow} \Gamma(V) \stackrel{P}{\longrightarrow} \Gamma(W) \longrightarrow 0 .
$$

This sequence is particularly useful to characterize the space of solutions to the equation $P \sigma=0$ for $\sigma \in \Gamma(V)$, that is to say $\operatorname{ker}(P)$, the kernel of $P$. As a matter of fact, (2-8) entails that $G$ induces an isomorphism from $\Gamma_{\mathrm{tc}}(W) / P\left(\Gamma_{\mathrm{tc}}(V)\right)$ to $\operatorname{ker}(P)$.

Following [Bär et al. 2007, Section 4.3], one can also relate Green operators over different globally hyperbolic spacetimes provided that the corresponding differential operators are related by vector bundle maps covering a causal embedding between the bases. Specifically, suppose we are given vector bundle maps $C: V_{1} \rightarrow V_{2}$ and $D: W_{1} \rightarrow W_{2}$ preserving the inner products of the relevant vector bundles and which cover a causal embedding $f: M_{1} \rightarrow M_{2}$ between globally hyperbolic spacetimes. Exploiting invertibility of vector bundle maps when restricted to a 
fiber, we can define maps between spaces of sections:

$$
\begin{array}{lll}
C^{\Gamma}: \Gamma\left(V_{2}\right) \rightarrow \Gamma\left(V_{1}\right), & \sigma_{2} \mapsto C^{-1} \circ \sigma_{2} \circ f, \\
C_{\Gamma_{\mathrm{c}}}: \Gamma_{\mathrm{c}}\left(V_{1}\right) \rightarrow \Gamma_{\mathrm{c}}\left(V_{2}\right), & \tau_{1} \mapsto C \circ \tau_{1} \circ f^{-1},
\end{array}
$$

and similarly for $D$. Furthermore, consider Green-hyperbolic differential operators $P_{i}: \Gamma\left(V_{i}\right) \rightarrow \Gamma\left(W_{i}\right), i \in\{1,2\}$, such that $P_{1} C^{\Gamma}=D^{\Gamma} P_{2}$. This simply means that $C$ and $D$ are compatible with the differential operators $P_{1}$ over $M_{1}$ and $P_{2}$ over $M_{2}$. Denoting the retarded/advanced Green operators for $P_{i}$ with $G_{i \pm}: \Gamma_{\mathrm{c}}\left(W_{i}\right) \rightarrow \Gamma\left(V_{i}\right)$, we can introduce $H_{1 \pm}=C^{\Gamma} G_{2 \pm} D_{\Gamma_{\mathrm{c}}}: \Gamma_{\mathrm{c}}\left(W_{1}\right) \rightarrow \Gamma\left(V_{1}\right)$ and compare it with $G_{1 \pm}$. Exploiting the fact that $f$ is a causal embedding, it is easy to check that $H_{1 \pm}$ fulfills the requirements in Definition 2.1, hence it is a retarded/advanced Green operator for $P_{1}$. By uniqueness, we conclude that $H_{1 \pm}=G_{1 \pm}$. Therefore, we have established a relation between $G_{1 \pm}$ and $G_{2 \pm}$, namely

$$
C^{\Gamma} G_{2 \pm} D_{\Gamma_{\mathrm{c}}}=G_{1 \pm}
$$

2C. General local covariance. We recall here the definition of a locally covariant quantum field theory according to [Brunetti et al. 2003] and briefly provide some motivation for this axiomatic approach to quantum field theory on curved spacetimes. This requires some basic notions coming from category theory, which can be found, e.g., in [MacLane 1971, Chapter 1].

We first introduce the relevant categories. As a source, we take a category GHyp having globally hyperbolic spacetimes $M$ as objects and causal embeddings $f: M \rightarrow N$ as morphisms; see Section 2A. This category provides the physical background where it is possible to sensibly discuss field theory, essentially because objects in this category possess a structure which is rich enough to make sense of initial value problems for hyperbolic partial differential equations, while morphisms are sufficiently well-behaved to allow us to relate Cauchy problems defined on different objects; see the end of Section 2B. The target category Alg is an algebraic one. Objects are unital $*$-algebras and morphisms are unit-preserving *-homomorphisms. Originally, morphisms in Alg where required to be injective, however we give up this assumption for reasons which will be clear later on. Objects in Alg are interpreted as the algebras of observables of a quantum field theory, while morphisms provide relations between different algebras arising from causal embeddings between globally hyperbolic spacetimes.

Definition 2.3. A locally covariant quantum field theory (LCQFT) is a functor $\mathscr{A}:$ GHyp $\rightarrow$ Alg fulfilling both causality and the time slice axiom.

Causality axiom: For each $f_{1}: M_{1} \rightarrow N$ and $f_{2}: M_{2} \rightarrow N$ in GHyp such that $f_{1}\left(M_{1}\right) \cap J_{N}\left(f_{2}\left(M_{2}\right)\right)=\varnothing$, we have $\left[\mathscr{A}\left(f_{1}\right) a_{1}, \mathscr{A}\left(f_{2}\right) a_{2}\right]=0$ for each $a_{1} \in$ $\mathscr{A}\left(M_{1}\right)$ and $a_{2} \in \mathscr{A}\left(M_{2}\right)$. 
Time slice axiom: For each $f: M \rightarrow N$ in GHyp such that $f(M)$ includes a spacelike Cauchy surface for $N, \mathscr{A}(f): \mathscr{A}(M) \rightarrow \mathscr{A}(N)$ is an isomorphism in Alg.

The functor $\mathscr{A}$ is interpreted in the following way: for each spacetime $M, \mathscr{A}$ assigns an algebra of observables $\mathscr{A}(M)$ defining the quantum field theory on $M$. Furthermore, whenever we have a causal embedding $f: M \rightarrow N, \mathscr{A}$ provides a *-homomorphism $\mathscr{A}(f)$ relating observables on the spacetime $M$ to their counterparts on $N$.

The original restriction to injective morphisms in Alg (not considered here) was meant to interpret globally hyperbolic subregions of a given spacetime as subsystems at the algebraic level (this property is often called isotony). Actually, even for those examples where the requirement of injectivity is violated [Dappiaggi and Lang 2012; Sanders et al. 2014; Benini et al. 2014a], one can recover the Haag-Kastler axioms [Haag and Kastler 1964; Dimock 1980; Benini et al. 2013] (and their interpretation in terms of subsystems) regarding a fixed spacetime as the full system and regions of this spacetime as subsystems; see [Benini et al. 2014a, Section 5].

As we will see later, injectivity does not hold in the case of the vector potential too. For this reason in the present context we refrain from requiring injectivity for the morphisms in Alg.

Causality entails that observables in causally disjoint regions can be tested independently. This condition implements the requirement that no physical information can propagate faster than light, hence a measurement localized in some region cannot affect other measurements which are localized in causally disjoint regions.

To conclude, the time slice axiom can be interpreted as a statement about the content of the algebra of observables on a given spacetime. It means that all observables on a given spacetime $N$ can be equivalently described by taking a globally hyperbolic neighborhood $M$ of any spacelike Cauchy surface in $N$. This behavior mimics the one of an initial value problem, where each solution is completely determined by its values in the vicinity of some spacelike Cauchy surface.

For the last part of this subsection we focus the attention on the relative Cauchy evolution. For a locally covariant quantum field theory, such a construction is made possible by the time slice axiom. The notion was introduced in [Brunetti et al. 2003, Section 4], where its relation with the quantized energy-momentum tensor was explicitly computed in the case of the Klein-Gordon field.

Suppose a locally covariant quantum field theory $\mathscr{A}:$ GHyp $\rightarrow$ Alg is given. Exploiting the time slice axiom, one can define the relative Cauchy evolution. We follow here the approach of [Fewster and Verch 2012, §3.4], where the construction presented below is described in full detail.

Given a globally hyperbolic spacetime $(M, g, \mathfrak{o}, \mathfrak{t})$, we introduce the set of hyperbolic perturbations $\mathrm{hp}(M)$ of $M$ as the set of compactly supported symmetric 
covariant 2-tensors $h$ on $M$ such that $g_{h}=g+h$ is a time-orientable Lorentzian metric on $M$ and $\left(M, g_{h}, \mathfrak{o}, \mathfrak{t}_{h}\right)$ is a globally hyperbolic spacetime, where $\mathfrak{t}_{h}$ is the unique time-orientation for $g_{h}$ agreeing with the original time-orientation $\mathfrak{t}$ outside $\operatorname{supp}(h)$.

Remark 2.4. As shown in [Beem et al. 1996, Section 7.1], $\mathrm{hp}(M)$ contains an open neighborhood of the zero section in the space of compactly supported covariant symmetric 2-tensors endowed with the test function topology. In particular it makes sense to endow $h p(M)$ with the topology induced as a subset of the space of compactly supported covariant symmetric 2-tensors.

Given a globally hyperbolic spacetime $M$ and a perturbation $h \in \mathrm{hp}(M)$, we indicate with $\tilde{M}$ the globally hyperbolic spacetime obtained perturbing the metric of $M$ as above. Denoting with $K$ the support of $h$, we introduce two globally hyperbolic spacetimes $M_{ \pm}=M \backslash J_{M}^{\mp}(K)$, which will act as intermediaries between $M$ and $\widetilde{M}$ at the algebraic level, making it possible to account for the effect of the metric perturbation $h$ on the space of observables $\mathscr{A}(M)$ associated to the original spacetime.

The construction proceeds observing that $M_{ \pm}$can be causally embedded in both $M$ and $\widetilde{M}$ according to the following diagram:

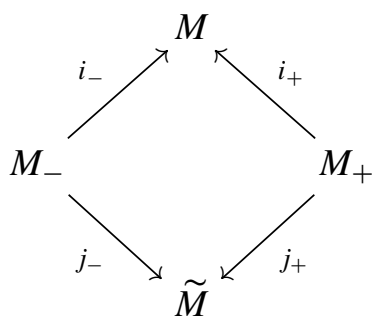

This construction is pictorially represented in Figure 1. Spacelike Cauchy surfaces for $M_{ \pm}$are spacelike Cauchy surfaces for $M$ and $\widetilde{M}$ too, as it can be checked directly from the definition of a Cauchy surface. Therefore the causal embeddings $i_{ \pm}$and $j_{ \pm}$fulfill the hypotheses in the statement of the time slice axiom, hence, applying the functor $\mathscr{A}$ to the diagram in (2-12), we get isomorphisms in Alg. This fact gives us the opportunity to define a special automorphism of $\mathscr{A}(M)$, namely the relative Cauchy evolution associated to the perturbation $h \in \mathrm{hp}(M)$ :

$$
R_{h}=\mathscr{A}\left(i_{-}\right) \mathscr{A}\left(j_{-}\right)^{-1} \mathscr{A}\left(j_{+}\right) \mathscr{A}\left(i_{+}\right)^{-1}: \mathscr{A}(M) \rightarrow \mathscr{A}(M) .
$$

$R_{h}$ is interpreted as the automorphic action induced by the metric perturbation $h$ on the space of observables $\mathscr{A}(M)$, which is assigned to the globally hyperbolic spacetime $M$.

In some sense, the relative Cauchy evolution provides the feedback at the level of observables induced by a modification of the metric localized in a compact region. 


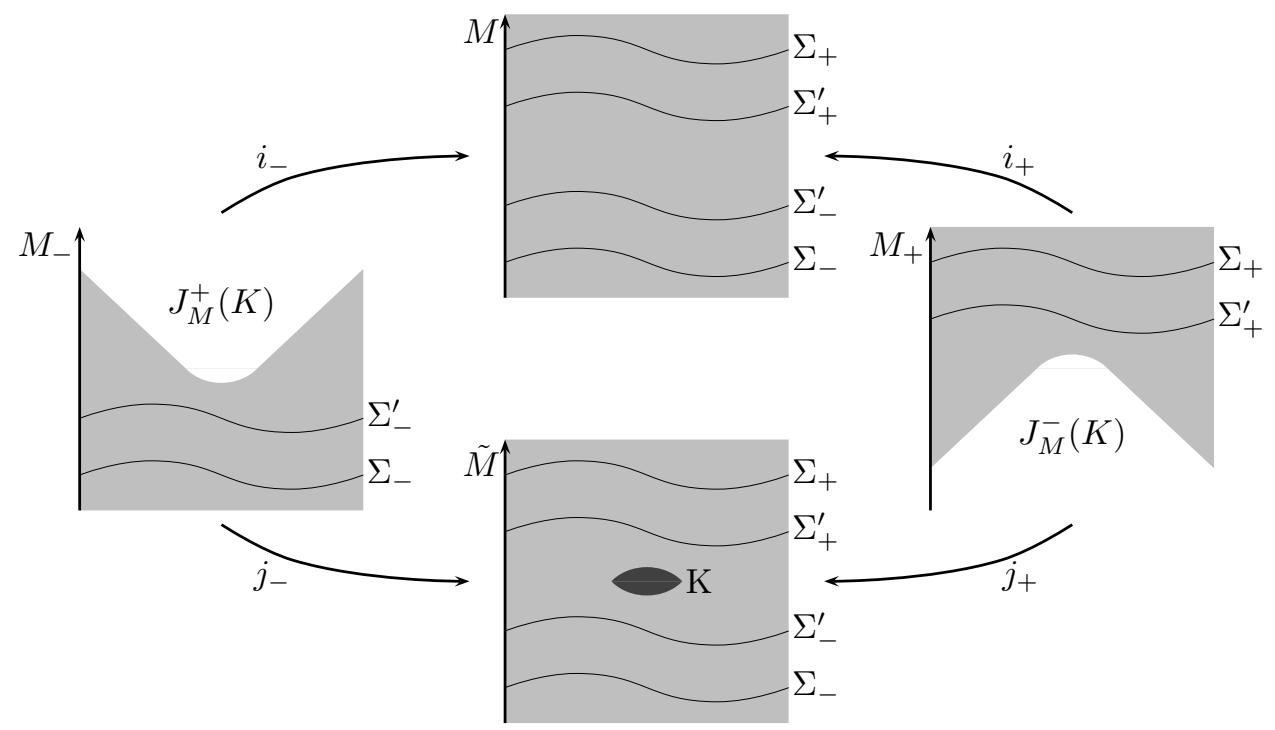

Figure 1. Pictorial representation of the globally hyperbolic spacetimes involved in the definition of the relative Cauchy evolution.

This is realized via $M_{ \pm}$in the following way. An observable on $M$ is mapped to $\tilde{M}$ via $M_{+}$exploiting the time slice axiom. In $\tilde{M}$ the observable is propagated through the region where the metric is perturbed, hence it is affected by the perturbation itself. This operation is performed in order to go back to the original spacetime $M$ via $M_{-}$, instead of following the same path in the opposite direction via $M_{+}$ (which leads to a trivial result). Once back to the unperturbed spacetime $M$, one can compare the original observable with the one given by the relative Cauchy evolution in order to evaluate the effect of the metric perturbation.

2D. *-algebras and states. As stated in the previous subsection, locally covariant quantum field theories are functors taking values in an appropriate category of *-algebras.

However, as we will see in the case of the vector potential, such a functor can be obtained quantizing a classical analogue of a locally covariant quantum field theory. This operation is performed introducing a quantization functor.

For the case we are interested in, we use a Alg-valued quantization functor 2 defined on the category PSym of presymplectic spaces. Objects of this category are pairs $(V, \sigma)$, where $V$ is a vector space and $\sigma$ is a presymplectic form on $V$, that is to say a (possibly degenerate) antisymmetric bilinear form on $V$; while morphisms $L:\left(V_{1}, \sigma_{1}\right) \rightarrow\left(V_{2}, \sigma_{2}\right)$ are linear maps $L: V_{1} \rightarrow V_{2}$ preserving the presymplectic structures $\sigma_{1}$ and $\sigma_{2}$, namely such that $\sigma_{2}\left(L v, L v^{\prime}\right)=\sigma_{1}\left(v, v^{\prime}\right)$ for each $v, v^{\prime} \in V_{1}$. 
Given a presymplectic space $(V, \sigma)$, we define a unital $*$-algebra $2(V, \sigma)$ using the Borchers-Uhlmann construction ([Borchers 1962; Uhlmann 1962]; see also [Brunetti et al. 2003, §2.6] or [Benini et al. 2014c, Appendix A]). To each element $v \in V$, we assign a hermitian symbol $\phi(v)=\phi(v)^{*}$. Then we take the unital $*-$ algebra $F$ freely generated over the field $\mathbb{C}$ by the symbols $\phi(v), v \in V$, and $\mathbb{1}$, the unit of the resulting algebra $F$. Singling out the $*$-ideal generated by elements of the form

$$
\begin{aligned}
\phi(a v+b w)-a \phi(v)-b \phi(w), & v, w \in V, a, b \in \mathbb{C}, \\
\phi(v) \phi(w)-\phi(w) \phi(v)-\mathrm{i} \sigma(v, w) \mathbb{1}, & v, w \in V,
\end{aligned}
$$

from the freely generated $*$-algebra $F$, we get $2(V, \sigma)$, the algebra of canonical commutation relations associated to the presymplectic space $(V, \sigma)$.

Remark 2.5. Note that (2-14) entails linearity of the implicitly defined quantization map

$$
\phi:(V, \sigma) \rightarrow 2(V, \sigma), \quad v \mapsto \phi(v),
$$

while (2-15) is used to enforce the usual canonical commutation relations for bosonic field theories, which is also the case for the vector potential of electromagnetism.

A morphism $L:\left(V_{1}, \sigma_{1}\right) \rightarrow\left(V_{2}, \sigma_{2}\right)$ induces a unit-preserving $*$-homomorphism at the level of the freely generated $*$-algebras. This is specified on generators by setting $\phi\left(v_{1}\right) \mapsto \phi\left(L v_{1}\right)$ for $v_{1} \in V_{1}$, and $\mathbb{1}_{1} \mapsto \mathbb{1}_{2}$. The obtained $*$-homomorphism naturally descends to the quotients by the $*$-ideals generated by (2-14) and (2-15); therefore we get a morphism $2(L): 2\left(V_{1}, \sigma_{1}\right) \rightarrow 2\left(V_{2}, \sigma_{2}\right)$ in Alg.

One can easily check that $2:$ PSym $\rightarrow$ Alg is a covariant functor, namely $2\left(\operatorname{id}_{(V, \sigma)}\right)=\operatorname{id}_{2(V, \sigma)}$ for each object $(V, \sigma)$ in PSym and $2\left(L^{\prime} L\right)=2\left(L^{\prime}\right) 2(L)$ for each pair of composable morphisms $L, L^{\prime}$ in PSym.

Remark 2.6. No topological information has been taken into account in the present construction. Actually, endowing a presymplectic space $(V, \sigma)$ with a topology (coherently, the presymplectic form $\sigma$ has to be continuous), one can consider on the $*$-algebra $2(V, \sigma)$ the topology induced by the construction above. The freely generated algebra $F$ is a direct limit; therefore it carries the topology canonically induced from that of $V$, thus becoming a topological $*$-algebra. That done, one should consider the quotient by the topological closure of the $*$-ideal generated by (2-14) and (2-15) in order to get a topological $*$-algebra $2(V, \sigma)$.

Let us mention that one might also adopt other quantization procedures, leading to much more regular algebras such as $C^{*}$-algebras. This works even in the case of arbitrary presymplectic groups; see [Manuceau et al. 1973]. 
A $*$-algebra is not enough for the physical description of a quantum field theory. One needs also an algebraic notion of state in order to evaluate the expectation value of an observable.

Definition 2.7. A state $\omega$ on a unital $*$-algebra $A$ is a normalized positive linear functional on $A$, namely $\omega: A \rightarrow \mathbb{C}$ is a linear map such that $\omega\left(a^{*} a\right) \geq 0$ for each $a \in A$ and $\omega(\mathbb{1})=1$.

A detailed analysis about algebraic states and their properties in relation to quantum field theory can be found, e.g., in [Bratteli and Robinson 1987; Bär et al. 2007; Bär and Becker 2009]. We would like to stress only one feature of algebraic states, that is the capability of reconstructing the usual Hilbert space representation of a quantum field theory exploiting the Gelfand-Naimark-Segal (GNS) theorem. Here we briefly recall this construction for a $*$-algebra without paying attention to topology. Some details for the case of topological $*$-algebras can be found in [Benini et al. 2013], while for the richer case of $C^{*}$-algebras see the references mentioned above.

Theorem 2.8. Let $A$ be $a *$-algebra and consider a state $\omega$ on A. Then there exist a Hilbert space $H$, a dense subspace $D \subseteq H$, a vector $\Omega \in D$ with norm 1 and $a *$-representation $\pi$ of $A$ by (possibly unbounded) linear maps on $H$ such that $\pi(A) \Omega=D$ and $\langle\Omega, \pi(\cdot) \Omega\rangle=\omega$, where $\langle\cdot, \cdot\rangle$ denotes the inner product on $H$. Moreover, the triple $(D, \pi, \Omega)$ with the properties mentioned above, called a GNS triple, is unique up to unitary equivalence.

Proof. It is possible to define a positive semidefinite sesquilinear form on $A$ (here regarded as a vector space only) exploiting positivity of the state:

$$
\langle\cdot, \cdot\rangle: A \times A \rightarrow \mathbb{C}, \quad(a, b) \mapsto \omega\left(b^{*} a\right) .
$$

Yet, $\langle\cdot, \cdot\rangle$ is degenerate if $N=\left\{a \in A: \omega\left(a^{*} a\right)=0\right\} \neq\{0\}$. Hermiticity follows from the fact that $\omega\left((a+\lambda b)^{*}(a+\lambda b)\right) \geq 0$ is a real number for each $a, b \in A$ and $\lambda \in \mathbb{C}$ (choose $\lambda=1$ and $\lambda=\mathrm{i}$ ). In particular, a Cauchy-Schwarz inequality for $\langle\cdot, \cdot\rangle$ can be established, namely $|\langle a, b\rangle|^{2} \leq\langle a, a\rangle\langle b, b\rangle$ for each $a, b \in A$. We deduce that $\langle a, a\rangle=0$ entails $\langle a, b\rangle=0$ for each $b \in A$. Therefore,

$$
N=\{a \in A:\langle a, b\rangle=0, \forall b \in A\}
$$

is a vector subspace of $A$ and we can consider the vector space $D=A / N$. By definition of $N$, the form $\langle\cdot, \cdot\rangle$ descends to the quotient $D$ as a positive definite sesquilinear form. Thus $D$ becomes a pre-Hilbert space. We denote its completion with $H$, which is a Hilbert space.

Notice that $N$ is left-invariant, namely $a N \subseteq N$ for each $a \in A$. This follows from $\langle a n, b\rangle=\omega\left(b^{*} a n\right)=\left\langle n, a^{*} b\right\rangle=0$ for each $a, b \in A$ and $n \in N$. This fact 
makes it possible to represent elements of $A$ by linear maps on $H$ :

$$
\pi: A \rightarrow L(H), \quad \pi(a)[b]=[a b], \forall a, b \in A .
$$

It is easy to check the identities $\pi(\mathbb{1})=\operatorname{id}_{H}$ and $\pi(a b)=\pi(a) \pi(b)$. Furthermore, $\left\langle\left[a^{*} b\right],[c]\right\rangle=\langle[b],[a c]\rangle$ entails that $\pi\left(a^{*}\right)$ defines the adjoint of $\pi(a)$, thus proving that $\pi$ is a $*$-representation of $A$ on $H$.

The unit $\mathbb{1} \in A$ defines a distinguished vector $\Omega=[\mathbb{1}] \in H$ of norm 1 , which allows us to reconstruct the algebraic state, $\langle\Omega, \pi(a) \Omega\rangle=\omega(a)$ for each $a \in A$. Moreover, $\pi(A) \Omega=D$ by definition.

Suppose that another triple $\left(D^{\prime}, \pi^{\prime}, \Omega^{\prime}\right)$ satisfying the same properties is given. We define $U: D \rightarrow D^{\prime}$ by $U \pi(a) \Omega=\pi^{\prime}(a) \Omega^{\prime}$ for each $a \in A$. The following identity, which holds true for each $a, b \in A$, entails that $U$ is well-defined as a linear map and preserves the scalar product:

$$
\left\langle\pi^{\prime}(a) \Omega^{\prime}, \pi^{\prime}(b) \Omega^{\prime}\right\rangle^{\prime}=\omega\left(b^{*} a\right)=\langle\pi(a) \Omega, \pi(b) \Omega\rangle .
$$

In particular, this entails that $U$ is bounded and thus it has a unique extension to the completions. In this way, we obtain $U: H \rightarrow H^{\prime}$, which is linear and continuous. Similarly, one can define $V: D^{\prime} \rightarrow D$ as $V \pi^{\prime}(a) \Omega^{\prime}=\pi(a) \Omega$ for each $a \in A$. Then, $V$ has the same properties as $U$. In particular, it preserves the scalar products and it admits a unique linear and continuous extension $V: H^{\prime} \rightarrow H$. From the definitions of $U$ and $V$, it is easy to check that $V$ is the inverse of $U$ on the dense subspaces $D$ and $D^{\prime}$; hence the same is true everywhere on $H$ and $H^{\prime}$. We conclude that $U: H \rightarrow H^{\prime}$ is a unitary equivalence such that $U \pi(\cdot)=\pi^{\prime}(\cdot) U$.

Remark 2.9. Even though the GNS construction can be carried out for $*$-algebras without any topology or taking into account a noncontinuous state, it turns out that operators representing elements of the $*$-algebra might be unbounded. This is not the case for more regular algebras (such as $C^{*}$-algebras) and continuous states.

Algebraic states provide the correct tool to evaluate expectation values of quantum observables. A quite large number of states is available for the algebra of a quantum field theory, yet not all of them exhibit a reasonable behavior from a physical perspective. A good criterion to select physically sensible states might be to mimic the properties shared by states for quantum field theories on Minkowski spacetime. Just to mention the most prominent states in this context, one encounters the vacuum, associated multi-particle states, coherent states and thermal equilibrium states as well. All these states share a peculiar behavior at very short distances, which plays a central role in the construction of Wick polynomials (which, in turn, provide an essential tool both to define physical quantities such as the quantized energy-momentum tensor and to discuss interacting models in a perturbative fashion). Therefore such short distance behavior seems to be vital for 
quantum field theory on Minkowski spacetime. An ultraviolet behavior of this kind is mathematically described by the so-called Hadamard condition. Fortunately, this condition on the high frequency part of the 2-point correlation function associated to a state has a natural counterpart on curved spacetimes, even though there is no coordinate independent notion of the Fourier transform. Seminal papers about this topic are [Radzikowski 1996a; 1996b], where tools from microlocal analysis [Hörmander 2003, Chapter 8] were employed in order to circumvent the lack of a good notion of Fourier transform. Since then, several techniques to construct Hadamard states on globally hyperbolic spacetimes for various field theoretical models were developed. We refer the reader to the very rich literature cited in [Benini et al. 2013, §4.3], where a concise review of the Hadamard condition, as well as a rich collection of distinguished examples of Hadamard states, can be found.

\section{Dynamics for the vector potential}

In this section we describe the classical field theory of the electromagnetic vector potential over a $d$-dimensional globally hyperbolic spacetime $M$. An approach similar to the one presented below can be found in [Fewster and Pfenning 2003; Dappiaggi 2011; Benini 2014].

The relevant vector bundles for this model are the exterior tensor powers $\wedge^{k} T^{*} M$ of the cotangent bundle $T^{*} M$. For each $k \in \mathbb{N}, \wedge^{k} T^{*} M$ can be canonically endowed with a nondegenerate inner product induced by the metric and the orientation of $M$. Denoting the exterior product with $\wedge: \wedge^{k} T^{*} M \times \wedge^{k^{\prime}} T^{*} M \rightarrow \wedge^{k+k^{\prime}} T^{*} M$ and introducing the Hodge dual $*: \wedge^{k} T^{*} M \rightarrow \bigwedge^{d-k} T^{*} M$ using the background metric $g$ and the orientation $\mathfrak{o}$, we get a nondegenerate inner product $\langle\cdot, \cdot\rangle=*^{-1}(\cdot \wedge * \cdot)$ on $\wedge^{k} T^{*} M$.

As is customary, we denote the space of sections of $\bigwedge^{k} T^{*} M$ (i.e., $k$-forms over $M)$ by $\Omega^{k}(M)=\Gamma\left(\bigwedge^{k} T^{*} M\right)$, The inner product $\langle\cdot, \cdot\rangle$ on the vector bundle $\wedge^{k} T^{*} M$, together with the volume form vol $=* 1$, defines an inner product $(\cdot, \cdot)$ on $k$-forms. Explicitly, we have

$$
(\alpha, \beta)=\int_{M} \alpha \wedge * \beta,
$$

for $\alpha, \beta \in \Omega^{k}(M)$ with compact overlapping supports.

On $k$-forms one has the differential d: $\Omega^{k}(M) \rightarrow \Omega^{k+1}(M)$ and the codifferential $\delta=(-1)^{k} *^{-1} \mathrm{~d} *: \Omega^{k}(M) \rightarrow \Omega^{k-1}(M)$. It is important to notice that $\mathrm{dd}=0$, hence $\delta \delta=0$, too. Moreover, one can directly check that $\delta$ is the formal adjoint of $\mathrm{d}$ with respect to $(\cdot, \cdot)$.

Using $\mathrm{d}$ and $\delta$, one can introduce the Laplace-de Rham operator $\square=\delta \mathrm{d}+\mathrm{d} \delta$ on $k$-forms, which is a differential operator of Green-hyperbolic type. It is easy 
to check that $\square$ is formally self-adjoint with respect to $(\cdot, \cdot)$ on account of the properties of $\mathrm{d}$ and $\delta$. This means that $\square^{*}=\square$, hence a similar relation holds true for the corresponding retarded/advanced Green operators, namely $G_{ \pm}^{*}=G_{ \pm}$. Therefore one also has

$$
\left(G_{+} \alpha, \beta\right)=\left(\alpha, G_{-} \beta\right)
$$

for each $k$-form $\alpha$ with past-compact support and each $k$-form $\beta$ with strictly futurecompact support. A similar result can be obtained by interchanging future and past. From dd $=0$ and $\delta \delta=0$, the identities for the Laplace-de Rham operator $\square$ and the Green operators $G_{ \pm}$follow:

$$
\begin{aligned}
\square \mathrm{d} & =\mathrm{d} \square, \quad \square \delta=\delta \square, \\
\mathrm{d} G_{ \pm} & =G_{ \pm} \mathrm{d}, \quad \delta G_{ \pm}=G_{ \pm} \delta,
\end{aligned}
$$

where we use the same symbols to denote the operators acting on forms of different rank. As an example, we show how to prove the first identity involving Green operators. Take any $\alpha \in \Omega_{\mathrm{pc}}^{k}(M)$ and any $\beta \in \Omega_{\mathrm{c}}^{k+1}(M)$ and compute $\left(\mathrm{d} G_{+} \alpha, \beta\right)$, exploiting the properties of the Green operators, formal self-adjointness of $\square$ as well as the identity $\square \mathrm{d}=\mathrm{d} \square$ :

$$
\begin{aligned}
\left(\mathrm{d} G_{+} \alpha, \beta\right) & =\left(\mathrm{d} G_{+} \alpha, \square G_{-} \beta\right)=\left(\square \mathrm{d} G_{+} \alpha, G_{-} \beta\right) \\
& =\left(\mathrm{d} \square G_{+} \alpha, G_{-} \beta\right)=\left(\mathrm{d} \alpha, G_{-} \beta\right)=\left(G_{+} \mathrm{d} \alpha, \beta\right) .
\end{aligned}
$$

Hence $\mathrm{d} G_{+}=G_{+} \mathrm{d}$, since $\alpha \in \Omega_{\mathrm{pc}}^{k}(M)$ and $\beta \in \Omega_{\mathrm{c}}^{k+1}(M)$ are arbitrary.

The Lagrangian density of electromagnetism $\mathscr{L}$ is expressed in terms of the Faraday tensor, $F \in \Omega^{2}(M)$ :

$$
\mathscr{L}=*\langle F, F\rangle=F \wedge * F .
$$

The Euler-Lagrange equations derived from $\mathscr{L}$ state that $F$ is a closed and coclosed 2-form; that is to say, $\mathrm{d} F=0$ and $\delta F=0$. In the following we consider only exact Faraday tensors, namely we assume there exists a vector potential $A \in \Omega^{1}(M)$ such that $\mathrm{d} A=F$. As a consequence, the first equation $\mathrm{d} F=0$ automatically holds true. The second equation remains to be checked, thus providing the dynamics of the vector potential, namely $\delta \mathrm{d} A=0$. Nevertheless, since the relevant object in electromagnetism is the Faraday tensor, we are forced to consider equivalence classes of vector potentials. As a matter of fact, two vector potentials $A$ and $A^{\prime}$ differing by $\mathrm{d} \varphi, \varphi \in \mathrm{C}^{\infty}(M)$, give rise to the same Faraday tensor $F=\mathrm{d} A$. For this reason, we consider gauge classes of vector potentials defined according to the equivalence relation

$$
A \sim A^{\prime} \Longleftrightarrow \exists \varphi \in \mathrm{C}^{\infty}(M) \text { such that } A^{\prime}=A+\mathrm{d} \varphi ;
$$


that is, $A, A^{\prime} \in \Omega^{1}(M)$ are considered the same whenever they differ by an exact 1 -form.

Let us mention that there are several other approaches to electromagnetism on curved spacetimes. This model was analyzed directly from the perspective of the Faraday tensor in [Dappiaggi and Lang 2012]. In [Dappiaggi and Siemssen 2013] and [Fewster and Lang 2014] the approaches are similar to the present one, except for the notion of gauge equivalence, which is provided there by closed 1-forms instead of exact ones. The present setting is adopted in [Sanders et al. 2014], where also external source currents are dealt with. Some arguments can be found there to motivate our choice of gauge symmetry (3-6). A more geometrical perspective, much in the spirit of Yang-Mills theory, can be found in [Benini et al. 2014b], subsequently refined in [Benini et al. 2014a], in order to correctly address the Aharonov-Bohm effect as well as magnetic monopoles.

In Section 2B we collected much of the material needed to characterize spaces of solutions for Green-hyperbolic equations in terms of Green operators. However this is not enough in the present setting for two reasons. First, the linear differential operator $\delta \mathrm{d}$ ruling the dynamics of the vector potential is not Green-hyperbolic. Second, we have to deal with gauge equivalence too. To overcome such hindrances, we are going to exploit gauge symmetry in order to show that equivalence classes of vector potentials satisfying the dynamics, $\delta \mathrm{d} A=0$, can be represented adopting the Lorenz gauge, that is to say $\delta A=0$. Later, exploiting the fact that $\square=\delta \mathrm{d}+\mathrm{d} \delta$ is Green-hyperbolic and realizing that on-shell vector potentials in the Lorenz gauge satisfy $\square A=0$, we provide a characterization of the space of gauge equivalence classes of solutions via the causal propagator $G=G_{+}-G_{-}$for $\square$, slightly extending a result in [Dappiaggi 2011]. A more systematic treatment of gauge theories can be found in [Hack and Schenkel 2013].

Lemma 3.1. Let $M$ be a globally hyperbolic spacetime. Denote the space of solutions to the equation ruling the dynamics of the vector potential by

$$
\mathscr{S}=\operatorname{ker}\left(\delta \mathrm{d}: \Omega^{1}(M) \rightarrow \Omega^{1}(M)\right) .
$$

Moreover, use $G=\mathrm{dC}^{\infty}(M)$ to denote the space of gauge transformations. Then for each gauge class of solutions $[A] \in \mathscr{Y} / \mathscr{G}$, there exists a representative $\hat{A} \in[A]$ in the Lorenz gauge; that is to say $\delta \hat{A}=0$.

Proof. Let $A \in[A]$ be any representative and consider the equation $\square \varphi+\delta A=0$ for $\varphi \in \mathrm{C}^{\infty}(M)$. We can easily write down a solution of this equation by fixing a partition of unity $\left\{\chi_{+}, \chi_{-}\right\}$such that $\chi_{ \pm}=1$ in a past- or future-compact region. With such a partition of unity, we get a solution $\varphi=-G_{+}\left(\chi_{+} \delta A\right)-G_{-}\left(\chi_{-} \delta A\right)$. Introducing $\hat{A}=A+\mathrm{d} \varphi$, we conclude that $\delta \mathrm{d} \hat{A}=0$ and $\delta \hat{A}=\delta A+\square \varphi=0$ since $\square=\delta \mathrm{d}$ on $\mathrm{C}^{\infty}(M)$. 
Since $\delta G_{ \pm}=G_{ \pm} \delta$ and taking into account (2-8) too, we realize that each coclosed $\alpha \in \Omega_{\mathrm{tc}}^{1}(M)$ gives rise to a solution $A=G \alpha \in \mathscr{Y}$ in the Lorenz gauge. This gives us a hint how to represent the space $\mathscr{T} / \mathscr{G}$ of gauge classes of solutions. Note that in the next proof we will extensively make use of the exact sequence (2-8).

Theorem 3.2. Let $M$ be a globally hyperbolic spacetime and set

$$
\operatorname{ker}_{\mathrm{tc}}^{k} \delta=\operatorname{ker}\left(\delta: \Omega_{\mathrm{tc}}^{k}(M) \rightarrow \Omega_{\mathrm{tc}}^{k-1}(M)\right) .
$$

Then the causal propagator $G$ for $\square=\delta \mathrm{d}+\mathrm{d} \delta$ induces the following isomorphism of vector spaces:

$$
I: \frac{\operatorname{ker}_{\mathrm{tc}}^{1}(\delta)}{\delta \mathrm{d}\left(\Omega_{\mathrm{tc}}^{1}(M)\right)} \rightarrow \frac{\mathscr{S}}{\mathscr{G}}, \quad[\alpha] \mapsto[G \alpha]
$$

Proof. As mentioned before the statement of the theorem, $G$ maps $\operatorname{ker}_{\mathrm{tc}}^{1}(\delta)$ to $\mathscr{S}$. Given $\beta \in \Omega_{\mathrm{tc}}^{1}(M), G \delta \mathrm{d} \beta=G(\square-\mathrm{d} \delta) \beta=\mathrm{d} G(-\delta \beta) \in \mathscr{G}$. Therefore $G$ induces a linear map from $\operatorname{ker}_{\mathrm{tc}}^{1}(\delta) / \delta \mathrm{d}\left(\Omega_{\mathrm{tc}}^{1}(M)\right)$ to $\mathscr{S} / \mathscr{G}$.

This map is surjective on account of Lemma 3.1. Given $[A] \in \mathscr{Y} / \mathscr{G}$, we find $\hat{A} \in[A]$ such that $\delta \hat{A}=0$. Since $\square \hat{A}=0$, using (2-8) we find $\hat{\alpha} \in \Omega_{\text {tc }}^{1}(M)$ such that $G \hat{\alpha}=\hat{A}$. From $\delta \hat{A}=0,(2-8)$ entails there exists $\psi \in \mathrm{C}_{\mathrm{tc}}^{\infty}(M)$ such that $\delta \hat{\alpha}=\square \psi=$ $\delta \mathrm{d} \psi$. We deduce that $\alpha=\hat{\alpha}-\mathrm{d} \psi \in \operatorname{ker}_{\text {tc }}^{1}(\delta)$. Moreover, $[G \alpha]=[\hat{A}-\mathrm{d} G \psi]=[A]$.

Given $\alpha \in \operatorname{ker}_{\mathrm{tc}}^{1}(\delta)$, it remains only to check that $[G \alpha]=0$ entails $\alpha \in \delta \mathrm{d}\left(\Omega_{\mathrm{tc}}^{1}(M)\right)$. By definition, we find $\varphi \in \mathrm{C}^{\infty}(M)$ such that $\mathrm{d} \varphi=G \alpha$, which entails $\square \varphi=0$. Therefore there exists $\psi \in \mathrm{C}_{\mathrm{tc}}^{\infty}(M)$ such that $G \psi=\varphi$. Hence (2-8) ensures the existence of $\beta \in \Omega_{\mathrm{tc}}^{1}(M)$ such that $\square \beta=\alpha-\mathrm{d} \psi$. Applying $\delta$ to both sides of the last identity, we get $\square \delta \beta=-\square \psi$, hence $\delta \beta=-\psi$. From this we conclude $\alpha=\delta \mathrm{d} \beta \in \delta \mathrm{d}\left(\Omega_{\mathrm{tc}}^{1}(M)\right)$.

Remark 3.3. One might be interested to solutions supported inside a spacelike compact region, namely consider $\mathscr{Y}_{\mathrm{sc}}=\left\{A \in \Omega_{\mathrm{sc}}^{1}(M): \delta \mathrm{d} A=0\right\}$. In this case the corresponding notion of gauge symmetry is specified by $\varphi_{\mathrm{sc}}=\mathrm{d} \Omega_{\mathrm{sc}}^{1}(M)$. Following the same arguments used above, but using the exact sequence (2-7) in place of (2-8), one gets an isomorphism of vector spaces similar to the one presented in Theorem 3.2:

$$
I_{s c}: \frac{\operatorname{ker}_{c}^{1}(\delta)}{\delta \mathrm{d}\left(\Omega_{\mathrm{c}}^{1}(M)\right)} \rightarrow \frac{\mathscr{\varphi}_{\mathrm{sc}}}{\mathscr{G}_{\mathrm{sc}}}, \quad[\alpha] \mapsto[G \alpha]
$$

where $\operatorname{ker}_{c}^{k}(\delta)=\operatorname{ker}\left(\delta: \Omega_{\mathrm{c}}^{k}(M) \rightarrow \Omega_{\mathrm{c}}^{k-1}(M)\right)$. In the next section we will encounter $\operatorname{ker}_{c}^{1}(\delta) / \delta \mathrm{d}\left(\Omega_{\mathrm{c}}^{1}(M)\right)$ (enriched with more structure) as the space of classical observables for the vector potential. Hence, via $I_{s c}$, one can interpret $\mathscr{S}_{\mathrm{sc}} / \mathscr{G}_{\mathrm{sc}}$ as the space of observables of the theory. This approach was adopted in [Dimock 1992; Pfenning 2009]. 


\section{Observables and quantization}

In this section we first introduce a suitable observables for the vector potential at a classical level. Then we quantize the obtained space of observables adopting the scheme presented in Section 2D.

In order to define observables for the vector potential, we follow the spirit of [Brunetti et al. 2012], where observables are defined as functionals on field configurations. In the case under analysis it is sufficient to take into account only linear functionals, the dynamics being linear. For this reason our approach mimics the one in [Benini et al. 2014c], even though the situation is even simpler, the underlying bundle being a vector bundle. For the first part of this section it is enough to consider $M$ to be a spacetime. When needed, we will also explicitly introduce the assumption of global hyperbolicity.

We start introducing a special class of linear functionals defined on the space of kinematically allowed field configurations, that is to say $\Omega^{1}(M)$ :

$$
\mathrm{O}_{\alpha}: \Omega^{1}(M) \rightarrow \mathbb{R}, \quad \mathrm{O}_{\alpha}(A)=(\alpha, A),
$$

where $\alpha \in \Omega_{\mathrm{c}}^{1}(M)$. We denote the space of such functionals with $\mathscr{E}^{\mathrm{kin}} \simeq \Omega_{\mathrm{c}}^{1}(M)$. The isomorphism $\alpha \mapsto \mathcal{O}_{\alpha}$ will be often used as an identification of $\mathscr{E}^{\mathrm{kin}}$ with $\Omega_{\mathrm{c}}^{1}(M)$. Since vector potentials differing by a gauge transformation are regarded to be equivalent, only gauge invariant functionals are relevant. For this reason we consider

$$
\mathscr{\mathscr { C }}^{\text {inv }}=\left\{\mathscr{O}_{\alpha} \in \mathscr{E}^{\mathrm{kin}}: \mathfrak{O}_{\alpha}(\mathrm{d} \varphi)=0, \forall \varphi \in \mathrm{C}^{\infty}(M)\right\} .
$$

Since $(\alpha, \mathrm{d} \varphi)=(\delta \alpha, \varphi)$ for each $\alpha \in \Omega_{\mathrm{c}}^{1}(M)$ and $\varphi \in \mathrm{C}^{\infty}(M)$, we conclude that $\mathscr{E}^{\text {inv }}=\operatorname{ker}_{c}^{1}(\delta)$. Up to now, no dynamical information is encoded in the space of gauge invariant functionals. As a matter of fact, $\mathscr{E}^{\text {inv }}$ provides gauge invariant functionals defined on all kinematically allowed field configurations, regardless of the equation of motion $\delta \mathrm{d} A=0$. In order to the encode dynamics in a dual fashion on gauge invariant functionals, we proceed as follows. First, we consider the formal adjoint of the equation of motion operator $\delta \mathrm{d}$, which is formally selfadjoint since $(\delta \mathrm{d} \alpha, A)=(\alpha, \delta \mathrm{d} A)$ for each $A \in \Omega^{1}(M)$ and each $\alpha \in \Omega_{\mathrm{c}}^{1}(M)$. Then, we take the quotient of $\mathscr{E}^{\text {inv }}$ by the image of $(\delta \mathrm{d})^{*}=\delta \mathrm{d}: \Omega_{\mathrm{c}}^{1}(M) \rightarrow \Omega_{\mathrm{c}}^{1}(M)$. In this way we obtain the vector space

$$
\mathscr{E}=\frac{\mathscr{E}^{\text {inv }}}{\delta \mathrm{d}\left(\Omega_{\mathrm{c}}^{1}(M)\right)},
$$

which is interpreted as a space of classical observables for the vector potential, the interpretation being motivated by the fact that $\mathscr{E}$ comprises gauge invariant functionals which can be evaluated only on on-shell field configurations $[A] \in \mathscr{S} / \mathscr{G}$. In fact, the evaluation of $[\alpha] \in \mathscr{E}$ on $[A] \in \mathscr{Y} / \mathscr{G}$ can be performed choosing arbitrary 
representatives in the equivalence classes. Such evaluation is well-defined because each $A \in[A]$ is on-shell, namely such that $\delta \mathrm{d} A=0$, and each $\alpha \in[\alpha]$ is gauge invariant.

The following theorem shows that $\mathscr{E}$ contains sufficiently many elements in order to distinguish vector potentials up to gauge. Moreover, if $M$ admits a finite good cover, there are no redundant elements of $\mathscr{E}$, namely two different elements cannot take the same values on all field configurations. Therefore $\mathscr{E}$ is optimal in the sense of [Benini 2014] as a space of linear classical observables for the model we are considering (under the assumption of existence of a finite good cover for the spacetime $M$ ). This result is a special case of [Benini 2014, Theorem 7.6]. For a different, yet equivalent, approach to causally restricted de Rham cohomology, see [Khavkine 2014a].

Theorem 4.1. Let $M$ be a spacetime and let $[\alpha],\left[\alpha^{\prime}\right] \in \mathscr{E}$ and $[A],\left[A^{\prime}\right] \in \mathscr{Y} / \mathscr{G}$.

(1) If $\mathscr{O}_{[\beta]}([A])=\mathscr{O}_{[\beta]}\left(\left[A^{\prime}\right]\right)$ for each $[\beta] \in \mathscr{E}$, then $[A]=\left[A^{\prime}\right]$.

(2) If $M$ admits a finite good cover and $O_{[\alpha]}([B])=O_{\left[\alpha^{\prime}\right]}([B])$ for each $[B] \in \mathscr{Y} / \mathscr{G}$, then $[\alpha]=\left[\alpha^{\prime}\right]$.

In view of our quantization prescription (see Section 2D) we want to endow $\mathscr{E}$ with a presymplectic structure. Assuming the spacetime $M$ to be globally hyperbolic and denoting the causal propagator for $\square$ with $G$, we define

$$
\tau: \mathscr{E} \times \mathscr{E} \rightarrow \mathbb{R}, \quad \tau([\alpha],[\beta])=(\alpha, G \beta),
$$

where $\alpha$ and $\beta$ are representatives of $[\alpha]$ and respectively $[\beta]$. The bilinear map $(\cdot, G \cdot): \Omega_{\mathrm{c}}^{1}(M) \times \Omega_{\mathrm{c}}^{1}(M) \rightarrow \mathbb{R}$ is antisymmetric:

$$
(\alpha, G \beta)=-(G \alpha, \beta)=-(\beta, G \alpha), \quad \forall \alpha, \beta \in \Omega_{\mathrm{c}}^{1}(M) .
$$

Moreover, for each $\alpha \in \operatorname{ker}_{c}^{1}(\delta)$ and $\omega \in \Omega_{\mathrm{c}}^{1}(M)$, one has

$$
(\alpha, G \delta \mathrm{d} \omega)=(\alpha, G(\square-\mathrm{d} \delta) \omega)=-(\delta \alpha, G \delta \omega)=0 .
$$

This shows that $\tau$ is well-defined by (4-1), thus providing a presymplectic form on $\mathscr{E}$.

Remark 4.2. The presymplectic form $\tau$ is actually degenerate on certain globally hyperbolic spacetimes. Suppose that $\beta$ lies in $\delta\left(\Omega_{\mathrm{c}}^{2}(M) \cap \mathrm{d} \Omega_{\mathrm{tc}}^{1}(M)\right) \backslash \delta \mathrm{d} \Omega_{\mathrm{c}}^{1}(M)$. This means that there exists $\gamma \in \Omega_{\mathrm{tc}}^{1}(M)$ such that $\delta \mathrm{d} \gamma=\beta$ and $\mathrm{d} \gamma$ has compact support, but there is no $\omega \in \Omega_{\mathrm{c}}^{1}(M)$ such that $\delta \mathrm{d} \omega=\beta$. Hence $[\beta] \neq 0$ in $\mathscr{E}$; however, by also exploiting (2-8), for each $[\alpha] \in \mathscr{E}$ we have

$$
\tau([\alpha],[\beta])=(\alpha, G \delta \mathrm{d} \gamma)=(\alpha, G(\square-\mathrm{d} \delta) \gamma)=-(\delta \alpha, G \delta \gamma)=0,
$$

where the last equality follows from $\delta \alpha=0$. 
Obviously, for a globally hyperbolic spacetime with compact Cauchy surfaces such an $\alpha$ cannot exist, timelike compact regions being compact too. However, it is relatively easy to cook up examples of globally hyperbolic spacetimes with noncompact Cauchy surfaces where $\delta\left(\Omega_{\mathrm{c}}^{2}(M) \cap \mathrm{d} \Omega_{\mathrm{tc}}^{1}(M)\right) \backslash \delta \mathrm{d} \Omega_{\mathrm{c}}^{1}(M)$ is not empty; see [Benini et al. 2014b, Remark 3.9].

Now we want to show that general local covariance (without injectivity) holds true for the field theoretical model considered here. This result will be achieved in two steps. First, we will construct a classical counterpart of a (noninjective) generally covariant quantum field theory for the vector potential. Then our quantization scheme will automatically provide a LCQFT according to Definition 2.3.

To each globally hyperbolic spacetime $M$ we assign the presymplectic space $\mathscr{F}(M)=\left(\mathscr{E}_{M}, \tau_{M}\right)$ as defined above (note that we included a subscript to keep track of the underlying spacetime). Given a causal embedding $f: M \rightarrow N$, we consider the pullback $f^{*}: \Omega^{k}(N) \rightarrow \Omega^{k}(M)$ for $k$-forms and the pushforward $f_{*}$ : $\Omega_{\mathrm{c}}^{k}(M) \rightarrow \Omega_{\mathrm{c}}^{k}(N)$ for compactly supported $k$-forms. $f_{*}$ intertwines the differential $\mathrm{d}_{M}$ for forms on $M$ with the differential $\mathrm{d}_{N}$ for forms on $N$, namely $f_{*} \mathrm{~d}_{M}=$ $\mathrm{d}_{N} f_{*}$. Moreover, since $f$ is an isometry, $f_{*} \delta_{M}=\delta_{N} f_{*}$ as well. Therefore $f_{*}$ induces a map $\mathscr{F}(f): \mathscr{E}_{M} \rightarrow \mathscr{E}_{N}$ between the spaces of observables associated to $M$ and $N$. It remains only to check that $\mathscr{F}(f)$ preserves the corresponding presymplectic structures $\tau_{M}$ and $\tau_{N}$. This follows from the last part of Section 2B. Taking into account the present setting, from Section 2B we deduce $f^{*} G_{N \pm} f_{*}=$ $G_{M \pm}$, where $G_{M \pm}$ and $G_{N \pm}$ denote the retarded/advanced Green operators for $\square_{M}$ and respectively $\square_{N}$. Given $[\alpha],[\beta] \in \mathscr{E}_{M}$, we compute

$$
\begin{aligned}
\tau_{N}(\mathscr{F}(f)[\alpha], \mathscr{F}(f)[\beta]) & =\left(f_{*} \alpha, G_{N} f_{*} \beta\right)_{N}=\left(\alpha, f^{*} G_{N} f_{*} \beta\right)_{M} \\
& =\left(\alpha, G_{M} \beta\right)_{M}=\tau_{M}([\alpha],[\beta]) .
\end{aligned}
$$

This shows that $\mathscr{F}(f): \mathscr{F}(M) \rightarrow \mathscr{F}(N)$ is a morphism in PSym. One can easily check that $\mathscr{F}_{(}\left(\mathrm{id}_{M}\right)=\mathrm{id}_{\mathscr{F}(M)}$ for each object $M$ in GHyp and that $\mathscr{F}\left(f \circ f^{\prime}\right)=$ $\mathscr{F}(f) \circ \mathscr{F}\left(f^{\prime}\right)$ for each pair of composable morphisms $f, f^{\prime}$ in GHyp. Therefore we conclude that $\mathscr{F}:$ GHyp $\rightarrow$ PSym is a functor.

Theorem 4.3. The functor $\mathscr{F}_{\mathrm{f}}:$ GHyp $\rightarrow$ PSym fulfills the classical counterparts of the causality and time slice axioms that are stated below. Yet injectivity of morphisms fails, namely there are morphisms $f$ in GHyp for which $\mathscr{F}(f)$ is not injective.

Causality axiom: For each $f_{1}: M_{1} \rightarrow N$ and $f_{2}: M_{2} \rightarrow N$ in GHyp such that $f_{1}\left(M_{1}\right) \cap J_{N}\left(f_{2}\left(M_{2}\right)\right)=\varnothing$, we have $\tau_{N}\left(\mathscr{F}\left(f_{1}\right)\left[\alpha_{1}\right], \mathscr{F}\left(f_{2}\right)\left[\alpha_{2}\right]\right)=0$ for each $\left[\alpha_{1}\right] \in \mathscr{F}\left(M_{1}\right)$ and $\left[\alpha_{2}\right] \in \mathscr{F}\left(M_{2}\right)$.

Time slice axiom: For each $f: M \rightarrow N$ in GHyp such that $f(M)$ includes a spacelike Cauchy surface for $N, \mathscr{F}(f): \mathscr{F}(M) \rightarrow \mathscr{F}(N)$ is an isomorphism in PSym. 
Proof. A counterexample to injectivity is provided in Remark 5.6 of [Benini et al. 2014b]. In fact, taking into account only the linear part of the classical observables defined in the reference just cited for the case $G=\mathbb{R}$ as structure group, one gets the same space of classical observables which is considered here.

The causality property is a trivial consequence of the support properties of the causal propagator. Under the assumptions of the statement, we have the inclusion

$$
\operatorname{supp}\left(f_{1 *} \alpha_{1}\right) \cap J_{N}\left(\operatorname{supp}\left(f_{2 *} \alpha_{2}\right)\right) \subseteq f_{1}\left(M_{1}\right) \cap J_{N}\left(f_{2}\left(M_{2}\right)\right)=\varnothing ;
$$

hence the supports of $f_{1 *} \alpha_{1}$ and $G_{N} f_{2 *} \alpha_{2}$ do not overlap. This shows that $\tau_{N}$ vanishes when evaluated on the pair $\left(\mathscr{F}\left(f_{1}\right)\left[\alpha_{1}\right], \mathscr{F}\left(f_{2}\right)\left[\alpha_{2}\right]\right) \in \mathscr{E}_{M} \times \mathscr{E}_{M}$.

To prove the time slice axiom we take $f: M \rightarrow N$ as in the statement and we look for an inverse of $\mathscr{F}(f)$.

As a preparatory step, we introduce a special partition of unity. Let $\Sigma$ be a spacelike Cauchy surface for $N$ included in $f(M)$. Since $f$ is a causal embedding, $f(M)$ is a globally hyperbolic spacetime with $\Sigma$ as a spacelike Cauchy surface. According to [Bernal and Sánchez 2005], we can foliate $N$ as $\mathbb{R} \times \Sigma$ and regard $f(M)$ as an open neighborhood of $\{0\} \times \Sigma$ in $N$. In particular, there are spacelike Cauchy surfaces $\Sigma_{+}, \Sigma_{-}$for $N$ of the form $\{t\} \times \Sigma$ which are contained in $f(M)$ and lie respectively inside the chronological future $I_{M}^{+}(\Sigma)$ and the chronological past $I_{M}^{-}(\Sigma)$ of $\Sigma$. We take a partition of unity $\left\{\chi_{+}, \chi_{-}\right\}$on $N$ such that $\chi_{ \pm}=1$ in $J_{N}^{ \pm}\left(\Sigma_{ \pm}\right)$

Using $\left\{\chi_{+}, \chi_{-}\right\}$, we define a map $I: \mathscr{E}_{N} \rightarrow \mathscr{E}_{M}$ according to the following procedure. Given $[\beta] \in \mathscr{E}_{N}$ and fixing a representative $\beta \in[\beta]$, we can consider the 1-form $\delta \mathrm{d}\left(\chi_{ \pm} G_{ \pm} \beta\right)=\beta-\delta \mathrm{d}\left(\chi_{\mp} G_{ \pm} \beta\right)$ and realize its support is compact. Here we exploited the compact support of $\beta$ and the past-compact/future-compact support of $\chi_{ \pm}$, together with $\delta \beta=0$ and Definition 2.1. Moreover, note that the left side vanishes in $J_{N}^{\mp}\left(\Sigma_{\mp}\right)$. We can also consider the 1 -form $\hat{\beta}=\delta \mathrm{d}\left(\chi_{+} G \beta\right)=$ $-\delta \mathrm{d}\left(\chi_{-} G \beta\right)$ (the second equality follows from $\delta \beta=0$ and $\left.\square G \beta=0\right)$. As it can be easily checked, $\hat{\beta}$ has compact support inside the time slab $J_{N}^{+}\left(\Sigma_{-}\right) \cap J_{N}^{-}\left(\Sigma_{+}\right) \subseteq$ $f(M)$. Setting $\omega=\left(\chi_{-} G_{+} \beta+\chi_{+} G_{-} \beta\right) \in \Omega_{\mathrm{c}}^{1}(N)$, by a direct computation we find $\hat{\beta}+\delta \mathrm{d} \omega=\beta$. Hence $\hat{\beta}$ is a representative of $[\beta]$ and, as we already proved, its support lies inside $f(M)$ allowing us to introduce $\alpha=f^{*} \hat{\beta} \in \Omega_{\mathrm{c}}^{1}(M)$ such that $\delta \alpha=0$. The same argument for another representative $\beta+\delta \mathrm{d} \gamma$ of $[\beta], \gamma \in \Omega_{\mathrm{c}}^{1}(M)$, would give $\hat{\beta}+\delta \mathrm{d}\left(\chi_{+} G \delta \mathrm{d} \gamma\right)$. Taking into account $G \square \gamma=0$, one gets

$$
\delta \mathrm{d}\left(\chi_{+} G \delta \mathrm{d} \gamma\right)=-\delta \mathrm{d}\left(\chi_{+} \mathrm{d} \delta G \gamma\right)=-\delta\left(\mathrm{d} \chi_{+} \wedge \mathrm{d} \delta G \gamma\right)=\delta \mathrm{d}\left(\mathrm{d} \chi_{+} \wedge \delta G \gamma\right),
$$

where $\hat{\gamma}=\mathrm{d} \chi_{+} \wedge \delta G \gamma$ has compact support in $J_{N}^{+}\left(\Sigma_{-}\right) \cap J_{N}^{-}\left(\Sigma_{+}\right) \subseteq f(M)$. This fact follows from $\mathrm{d} \chi_{+}=-\mathrm{d} \chi_{-}$being supported inside a timelike compact region and $\operatorname{supp}(G \omega)$ being spacelike compact. Introducing $\theta=f^{*} \hat{\gamma} \in \Omega_{\mathrm{c}}^{1}(M)$, we conclude that, when starting from $\beta+\delta \mathrm{d} \gamma$, the procedure above provides $\alpha+\delta \mathrm{d} \theta$. 
Since $[\alpha+\delta \mathrm{d} \theta]=[\alpha]$ in $\mathscr{E}_{M}$, we can define the linear map

$$
I: \mathscr{E}_{N} \rightarrow \mathscr{E}_{M}, \quad[\beta] \mapsto\left[f^{*} \delta \mathrm{d}\left(\chi_{+} G \beta\right)\right] .
$$

One can directly check that $I$ is actually the inverse of $\mathscr{F}(f)$ taking $[\alpha] \in \mathscr{E}_{M}$ and $[\beta] \in \mathscr{E}_{N}$ and computing $I \mathscr{F}(f)[\alpha]$ and $\mathscr{F}(f) I[\beta]$. In the first formula below we introduce subscripts on $G$ to stress that both the causal propagators for $\square$ on $M$ and for $\square$ on $N$ are involved.

$$
\begin{aligned}
I \mathscr{F}(f)[\alpha] & =I\left[f_{*} \alpha\right]=\left[f^{*} \delta \mathrm{d}\left(\chi_{+} G_{N} f_{*} \alpha\right)\right]=\left[\delta \mathrm{d}\left(\left(f^{*} \chi_{+}\right) G_{M} \alpha\right)\right] \\
& =\left[\delta \mathrm{d}\left(\left(f^{*} \chi_{+}\right) G_{M} \alpha\right)+\delta \mathrm{d}\left(\left(f^{*} \chi_{+}\right) G_{M-} \alpha\right)+\delta \mathrm{d}\left(\left(f^{*} \chi_{-}\right) G_{M+} \alpha\right)\right] \\
& =\left[\delta \mathrm{d}\left(G_{M+} \alpha\right)\right]=[\alpha], \\
\mathscr{F}(f) I[\beta] & =\mathscr{F}(f)\left[f^{*} \delta \mathrm{d}\left(\chi_{+} G \beta\right)\right]=\left[\delta \mathrm{d}\left(\chi_{+} G \beta\right)\right]=[\beta] .
\end{aligned}
$$

For the first computation we exploited the fact that $\left(f^{*} \chi_{ \pm}\right) G_{M \mp} \alpha$ is a compactly supported 1-form on $M$, while the second follows from the fact that $\delta \mathrm{d}\left(\chi_{+} G \beta\right)$ is a representative of $[\beta]$ with support inside $f(M)$, as already shown above. Automatically $I$ preserves the relevant presymplectic forms:

$$
\tau_{M}\left(I[\beta], I\left[\beta^{\prime}\right]\right)=\tau_{N}\left(\mathscr{F}(f) I[\beta], \mathscr{F}(f) I\left[\beta^{\prime}\right]\right)=\tau_{N}\left([\beta],\left[\beta^{\prime}\right]\right),
$$

for each $[\beta],\left[\beta^{\prime}\right] \in \mathscr{E}_{N}$. This shows that $I: \mathscr{F}(N) \rightarrow \mathscr{F}(M)$ is the inverse of $\mathscr{F}(f): \mathscr{F}(M) \rightarrow \mathscr{F}(N)$ in PSym; therefore $\mathscr{F}(f)$ is an isomorphism in PSym.

The last part of this section is devoted to the quantization of the functor $\mathscr{F}$ describing the classical field theory of the vector potential. This result is achieved composing $\mathscr{F}:$ GHyp $\rightarrow$ PSym with the quantization functor $2:$ PSym $\rightarrow$ Alg for canonical commutation relations presented in Section 2D.

Theorem 4.4. The functor $\mathscr{A}=2 \circ \mathscr{F}: \mathrm{GHyp} \rightarrow \mathrm{Alg}$ is a locally covariant quantum field theory according to Definition 2.3.

Proof. $\mathscr{A}$ is defined by the composition of the covariant functors $2:$ PSym $\rightarrow$ Alg and $\mathscr{F}:$ GHyp $\rightarrow$ PSym; therefore it is a covariant functor from GHyp to Alg. Causality holds true on account of its classical counterpart fulfilled by $\mathscr{F}$ and the canonical commutation relations implemented by 2 . The time slice axiom for $\mathscr{A}$ follows from the corresponding property of $\mathscr{F}$ and the fact that 2 is a functor, thus sending isomorphisms of PSym to isomorphisms of Alg.

Remark 4.5. Up to now, neither $\mathscr{F}(M)$ nor $\mathscr{A}(M)$ were intended as topological spaces. Actually, one can endow $\mathscr{F}(M)$ with the topology induced by the test function topology on $\Omega_{\mathrm{c}}^{1}(M), \mathscr{F}(M)$ being the quotient by $\delta \mathrm{d}\left(\Omega_{\mathrm{c}}^{1}(M)\right)$ of the closed subspace $\mathscr{E}^{\text {inv }}$ of $\Omega_{\mathrm{c}}^{1}(M)$. At least whenever $M$ admits a finite good cover, the second statement of Theorem 4.1 means that the image of $\delta \mathrm{d}: \Omega_{\mathrm{c}}^{1}(M) \rightarrow \Omega_{\mathrm{c}}^{1}(M)$ coincides with the intersection of the kernels of the maps $\int_{M}(\cdot) \wedge * A: \Omega_{\mathrm{c}}^{1}(M) \rightarrow \mathbb{R}$, 
$A \in \operatorname{ker}^{1}(\delta \mathrm{d})$. Since these maps are continuous, $\delta \mathrm{d}\left(\Omega_{\mathrm{c}}^{1}(M)\right)$ is a closed subspace of $\Omega_{\mathrm{c}}^{1}(M)$. This entails that the topology induced on $\mathscr{F}(M)$ by the quotient is Hausdorff at least when $M$ admits a finite good cover. Continuity of the push-forward along a smooth map and of the Green functions (see [Bär et al. 2007, Section 3.4]) entails that all our conclusions up to this point are compatible with the topological structure presented above, in particular $\mathscr{F}$ is a functor taking values in the category of topological presymplectic spaces. Therefore, Remark 2.6 entails that the functor $A$ can be regarded as taking values in the category of unital topological $*$-algebras.

Theorem 4.4 provides a satisfactory description of the quantum field theory of the vector potential on each globally hyperbolic spacetime. One still needs Hadamard states for this model. A constructive result in this direction can be found in [Dappiaggi and Siemssen 2013] for asymptotically flat globally hyperbolic spacetimes at future null infinity. Furthermore, the existence of Hadamard states can be argued exploiting a deformation arguments involving ultrastatic spacetimes [Fulling et al. 1978; Fulling et al. 1981], where a complete timelike Killing vector field makes it possible to cook up Hadamard states by means of Fourier transform techniques. This approach was followed in [Fewster and Pfenning 2003]. An extension of the Gupta-Bleuler formalism to curved spacetimes is available too; see [Finster and Strohmaier 2013].

We recall the notion of a quasifree Hadamard state for the vector potential according to [Fewster and Pfenning 2003].

Definition 4.6. For a globally hyperbolic spacetime $M$, a state $\omega$ on the field algebra $\mathscr{A}(M)$ is quasifree and Hadamard if there exists a distribution $w \in \Omega_{\mathrm{c}}^{1}\left(M^{2}\right)^{\prime}$ fulfilling the requirements listed below.

(1) $w$ is a $\square$-bisolution, i.e., $w(\square \alpha, \beta)=0=w(\alpha, \square \beta)$ for each $\alpha, \beta \in \Omega_{\mathrm{c}}^{1}(M)$.

(2) $w(\alpha, \beta)-w(\beta, \alpha)=i \tau([\alpha],[\beta])$ for each $\alpha, \beta \in \mathscr{E}^{\text {inv }}$.

(3) The wavefront set of $w$ has the form

$$
W F(w)=\left\{\left(x, k ; x^{\prime},-k^{\prime}\right) \in \dot{T}^{*} M^{2}:(x, k) \sim\left(x^{\prime}, k^{\prime}\right), k \in V_{x}^{+}\right\},
$$

where $\dot{T}^{*}$ denotes the cotangent bundle with the zero section removed, $V_{x}^{+}$is the closed cone of lightlike covectors at $x$ and $(x, k) \sim\left(x^{\prime}, k^{\prime}\right)$ means that $x$ is joined to $x^{\prime}$ by a lightlike geodesic $\gamma, k$ is the cotangent vector at $x$ of $\gamma$ and $k^{\prime}$ is the parallel transport of $k$ along $\gamma$.

(4) The two-point function of the state $\omega$ is given by $w$, that is to say, for each $\alpha, \beta \in \mathscr{E}^{\text {inv }}$,

$$
\omega(\phi([\alpha]) \phi([\beta]))=w(\alpha, \beta) .
$$


(5) All $n$-point functions vanish for $n$ odd, while for $n$ even they are completely determined by two-point function, namely, for $\alpha_{1}, \ldots, \alpha_{n} \in \mathscr{E}$ Einv

$$
\omega\left(\phi\left(\left[\alpha_{1}\right]\right) \cdots \phi\left(\left[\alpha_{n}\right]\right)\right)=\sum_{\pi \in P_{n}} \prod_{i=1}^{n / 2} \omega\left(\phi\left(\left[\alpha_{\pi(2 i-1)}\right]\right) \phi\left(\left[\alpha_{\pi(2 i)}\right]\right)\right),
$$

where $P_{n}$ denotes the set of permutations $\pi$ of $\{1, \ldots, n\}$ such that $\pi(2 i-1)<$ $\pi(2 i+1)$ and $\pi(2 i-1)<\pi(2 i)$ for each $i \in\{1, \ldots, n / 2\}$.

\section{Relative Cauchy evolution}

In this section we relate the relative Cauchy evolution to the energy-momentum tensor of the vector potential, thus extending a result which was originally established for the Klein-Gordon field in [Brunetti et al. 2003] and later shown to hold in the Dirac case as well; see [Sanders 2010].

As a starting point, we fix a globally hyperbolic spacetime $M$ and we consider a representation $\pi$ of the field algebra $\mathscr{A}(M)$ (meant here as a unital topological *-algebra according to Remark 4.5) on a Hilbert space $H$ such that it makes sense to consider the functional derivative of the relative Cauchy evolution $R_{h}$ with respect to the perturbation $h$. This is to be intended in the following sense: there exists a dense subspace $S$ of $H$ and a dense unital $*$-subalgebra $B$ of $\mathscr{A}(M)$ such that, for each $\theta \in S$ and $b \in B$, there exists a symmetric contravariant 2-tensor $t$ satisfying the following condition:

$$
\int_{M}\left(\left.\frac{\mathrm{d}}{\mathrm{d} s}\right|_{0} h_{s \mu \nu}\right) t^{\mu \nu} \mathrm{vol}=\left.\frac{\mathrm{d}}{\mathrm{d} s}\right|_{0}\left|\theta, \pi\left(R_{s} b\right) \theta\right\rangle,
$$

for each compact set $K \subseteq M$ and each smooth 1-parameter family $s \in(-1,1) \mapsto$ $h_{s} \in \mathrm{hp}(M)$ of globally hyperbolic perturbations of $M$ with support inside $K$, where $R_{s}$ stands for $R_{h_{s}} \cdot{ }^{2}$ Uniqueness of $t$ follows from Remark 2.4. This allows us to introduce

$$
(\theta, b) \in S \times B \mapsto\left\langle\theta,\left(\frac{\delta}{\delta h} \pi\left(R_{h} b\right)\right) \theta\right\rangle \doteq t,
$$

which implicitly defines, for each $b \in B$, the functional derivative $\delta \pi\left(R_{h} b\right) / \delta h$ of the relative Cauchy evolution as a quadratic form on $V$ via the representation $\pi$.

As noted in [Brunetti et al. 2003], the GNS representation $\pi_{\omega}$ induced by a quasifree Hadamard state $\omega$ on the field algebra $\mathscr{A}(M)$ fulfills all requirements listed above for defining the functional derivative of the relative Cauchy evolution.

Remark 5.1. Using general arguments, in [Brunetti et al. 2003, Theorem 4.2] it is shown that $\delta \pi\left(R_{h} b\right) / \delta h$ is divergence-free with respect to the Levi-Civita

\footnotetext{
${ }^{2}$ We will use this notation whenever it is clear from the context which family of perturbations is being taken into account.
} 
connection for the unperturbed metric $g$. This is a consistency check for the main theorem of this section since the final result consists of an equality between the functional derivative of the relative Cauchy evolution and a term involving the energy-momentum tensor of the electromagnetic field, which is divergence-free.

For convenience, we first state the final result and then we proceed step by step preparing the tools needed later for the proof.

Theorem 5.2. Let $M$ be a globally hyperbolic spacetime and consider a quasifree Hadamard state $\omega$ for the field algebra $A(M)$ of the vector potential. Consider the GNS triple $\left(D_{\omega}, \pi_{\omega}, \Omega_{\omega}\right)$ associated to $\omega$. Then the equality stated below holds true for each $[\alpha] \in \mathscr{F}(M)$ in the sense of quadratic forms on $D_{\omega}$ :

$$
\frac{\delta}{\delta h_{\mu \nu}} \pi_{\omega}\left(R_{h} \phi([\alpha])\right)=\frac{\mathrm{i}}{2}\left[\hat{T}^{\mu \nu}, \phi_{\omega}([\alpha])\right],
$$

where $\phi_{\omega}([\alpha])=\pi_{\omega}(\phi([\alpha]))$ is a generator of the field algebra $A(M)$ represented via $\pi_{\omega}$ and $\hat{T}^{\mu v}$ is the quantized energy-momentum tensor (indices are raised using the background metric) obtained via point-splitting in the GNS representation induced by $\omega$.

Remark 5.3. Instead of using the point-splitting prescription in order to quantize the classical energy-momentum tensor $T_{\mu \nu}$ of the electromagnetic field, defined as the functional derivative of the action with respect to the background metric, one could consider more refined quantization procedures, but the conclusions of Theorem 5.2 would not be affected. For details see the remarks after [Brunetti et al. 2003, Theorem 4.3].

5A. Energy-momentum tensor and point-splitting. As a starting point, we write down the classical energy-momentum tensor as the functional derivative with respect to the spacetime metric of the action $S$ for the vector potential $A$, which is defined out of the Lagrangian (3-5):

$$
T_{\mu \nu}=\frac{2}{\sqrt{|\operatorname{det} g|}} \frac{\delta S}{\delta g^{\mu \nu}}=F_{\mu \rho} F_{\nu}{ }^{\rho}-\frac{1}{4} g_{\mu \nu} F_{\rho \sigma} F^{\rho \sigma},
$$

where indices are raised with respect to the spacetime metric $g$. The result is a divergence-free symmetric covariant 2-tensor $T$, as one can easily check taking into account the identities $F=\mathrm{d} A$ and $\delta \mathrm{d} A=0$.

In Theorem 5.2 the quantized energy-momentum tensor $\hat{T}$ appears. This is obtained from the classical one, namely $T$, applying the point-splitting prescription [Wald 1994, Section 4.6]:

(1) Separate products of classical fields at the same spacetime point $p \in M$ introducing an auxiliary base point $q \in M$. This is to be intended in the limit 
where the two points are close enough. To be more precise, one should consider a point $q \neq p$ in a normal neighborhood of $p$. This way there exists a unique geodesic connecting $p$ to $q$. The product at different spacetime points is then properly defined in terms of the parallel transport operator $Y$ along such geodesic;

(2) Replace products of classical fields with matrix elements of products of quantum fields in a Hilbert space representation;

(3) Deal with all computations in the point-split form. Only in the end take the coincidence limit, provided no singularity arises.

To start with, one has to define matrix elements for products of quantum fields in the GNS representation $\pi_{\omega}$ induced by a quasifree Hadamard state $\omega$. According to Definition 4.6, we note that, given $\xi, \eta \in D_{\omega}$ and $\alpha_{1}, \ldots, \alpha_{n} \in \mathscr{E}^{\text {inv }}$, the matrix element of a product of $n$ fields $\left\langle\xi, \phi_{\omega}\left(\left[\alpha_{1}\right]\right) \cdots \phi_{\omega}\left(\left[\alpha_{n}\right]\right) \eta\right\rangle$ can be written as a sum of products of a suitable bidistribution $w$ evaluated on some test-sections in $\mathscr{E}^{\text {inv }}$, among which one finds $\alpha_{1}, \ldots, \alpha_{n}$. Therefore, using $w$, one can define a $n$ distribution $\left\langle\xi, \hat{A}\left(p_{1}\right) \cdots \hat{A}\left(p_{n}\right) \eta\right\rangle \in \Omega_{\mathrm{c}}^{1}\left(M^{n}\right)^{\prime}$ satisfying, for each $\alpha_{1}, \ldots, \alpha_{n} \in \mathscr{C}^{\mathrm{inv}}$, the identity

$$
\begin{aligned}
\int_{M^{n}}\left\langle\xi, \hat{A}_{\mu_{1}}\left(p_{1}\right) \cdots \hat{A}_{\mu_{n}}\left(p_{n}\right) \eta\right\rangle \alpha_{1}^{\mu_{1}}\left(p_{1}\right) \cdots \alpha_{n}^{\mu_{n}}\left(p_{n}\right) \text { vol } & \\
& =\left\langle\xi, \phi_{\omega}\left(\left[\alpha_{1}\right]\right) \cdots \phi_{\omega}\left(\left[\alpha_{n}\right]\right) \eta\right\rangle,
\end{aligned}
$$

where the integral denotes evaluation of a distribution on a test section and indices are raised with respect to $g$.

However, $\left\langle\xi, \hat{A}\left(p_{1}\right) \cdots \hat{A}\left(p_{n}\right) \eta\right\rangle$ is not the only distribution satisfying (5-4). Since only elements of $\mathscr{E}^{\text {inv }} \subseteq \Omega_{\mathrm{c}}^{1}(M)$ can enter $\left\langle\xi, \phi_{\omega}\left(\left[\alpha_{1}\right]\right) \cdots \phi_{\omega}\left(\left[\alpha_{n}\right]\right) \eta\right\rangle$, one can add exact $n$-distributions to $\left\langle\xi, \hat{A}\left(p_{1}\right) \cdots \hat{A}\left(p_{n}\right) \eta\right\rangle$ without affecting the identity (5-4). This ambiguity does not affect the quantization of $T$ since only $F=\mathrm{d} A$ enters (5-3), and therefore Theorem 5.2 is not affected as well.

Remark 5.4. For $n=1,\left\langle\xi, \hat{A}_{\mu}(p) \eta\right\rangle$ is a distribution generated by a smooth function, as observed in [Brunetti et al. 2003, p. 60].

Using (5-4), from items (1)-(2) above we get matrix elements for the quantized energy-momentum tensor:

$\left\langle\xi, \hat{T}_{\mu \nu}(p, q) \eta\right\rangle$

$$
\begin{aligned}
& =g^{\rho \sigma}(p) Y_{v \sigma}^{v^{\prime} \sigma^{\prime}}(p, q) \\
& \quad \times\left[\nabla_{\mu}^{p} \nabla_{v^{\prime}}^{q}\left(\xi, \hat{A}_{\rho}(p) \hat{A}_{\sigma^{\prime}}(q) \eta\right\rangle-\left(v^{\prime} \leftrightarrow \sigma^{\prime}\right)-(\mu \leftrightarrow \rho)+\left(\mu \leftrightarrow \rho \text { and } v^{\prime} \leftrightarrow \sigma^{\prime}\right)\right] \\
& -\frac{1}{2} g_{\mu \nu}(p) g^{\rho \sigma}(p) g^{\tau v}(p) Y_{\sigma v}^{\sigma^{\prime} v^{\prime}}(p, q) \\
& \quad \times\left[\nabla_{\rho}^{p} \nabla_{\sigma^{\prime}}^{q}\left\langle\xi, \hat{A}_{\tau}(p) \hat{A}_{v^{\prime}}(q) \eta\right\rangle-\left(\sigma^{\prime} \leftrightarrow v^{\prime}\right)\right] . \quad \text { (5-5) }
\end{aligned}
$$


Here the superscript on a covariant derivative indicates the spacetime dependence of the section upon which the covariant derivative is applied; $\mu \leftrightarrow \rho$ stands for a term equal to the one explicitly written before, with indices $\mu$ and $\rho$ interchanged. Setting $\eta=\phi_{\omega}([\alpha]) \theta, \xi=\theta$ and then $\xi=\theta, \eta=\phi_{\omega}([\alpha]) \theta$ in (5-5) and taking the difference between the two outcomes, one gets $\left\langle\theta,\left[\hat{T}_{\mu \nu}(p, q), \phi_{\omega}([\alpha])\right] \theta\right\rangle$, which is the relevant term for Theorem 5.2. A closer look at (5-5) shows that this term can be evaluated once $\left\langle\theta,\left[\hat{A}_{\mu}(p) \hat{A}_{v^{\prime}}(q), \phi_{\omega}([\alpha])\right] \theta\right\rangle$ is known. This can be obtained from the term $\left\langle\theta,\left[\phi_{\omega}([\beta]) \phi_{\omega}([\gamma]), \phi_{\omega}([\alpha])\right] \theta\right\rangle$ by extracting a (nonunique) bidistribution as above. Using the canonical commutation relations (2-15), we obtain

$$
\left\langle\theta,\left[\hat{A}_{\mu}(p) \hat{A}_{\nu^{\prime}}(q), \phi_{\omega}([\alpha])\right] \theta\right\rangle=\mathrm{i}(G \alpha)_{\mu}(p)\left\langle\theta, \hat{A}_{v^{\prime}}(q) \theta\right\rangle+\mathrm{i}(G \alpha)_{\nu^{\prime}}(q)\left\langle\theta, \hat{A}_{\mu}(p) \theta\right\rangle .
$$

As above, the one on the right side is not the only possible choice of a bidistribution representing the left side, yet this ambiguity amounts to an exact bidistribution, and therefore it disappears as soon as we take the appropriate derivatives in order to evaluate $\left\langle\theta,\left[\hat{T}_{\mu \nu}(p, q), \phi_{\omega}([\alpha])\right] \theta\right\rangle$. Already at this stage one realizes no singularity appears in the coincidence limit $p \rightarrow q$. Defining $A=G \alpha, F=\mathrm{d} A, \tilde{A}=\langle\theta, \hat{A} \theta\rangle$ and $\tilde{F}=\mathrm{d} \tilde{A}$, one is led to

$$
\begin{aligned}
\left\langle\theta,\left[\hat{T}_{\mu \nu}(p), \phi_{\omega}([\alpha])\right] \theta\right\rangle=\mathrm{i} g^{\rho \sigma}(p) & \left(F_{\mu \rho}(p) \tilde{F}_{\nu \sigma}(p)+\tilde{F}_{\mu \rho}(p) F_{\nu \sigma}(p)\right) \\
& -\frac{\mathrm{i}}{2} g_{\mu \nu}(p) g^{\rho \sigma}(p) g^{\tau v}(p) F_{\rho \tau}(p) \tilde{F}_{\sigma v}(p) .
\end{aligned}
$$

5B. Classical relative Cauchy evolution. This subsection is devoted to finding a convenient formula for the relative Cauchy evolution at the classical level. This is defined replacing $\mathscr{A}$ in (2-13) with $\mathscr{F}$, the functor describing the classical field theory of the vector potential. This can be done on account of the time slice axiom Theorem 4.3. In view of the proof of Theorem 5.2, given a globally hyperbolic spacetime $M$, we fix a compact region $K \subseteq M$ and a 1-parameter family $s \in(-1,1) \mapsto h_{s} \in \mathrm{hp}(M)$ supported inside $K$. For each $s \in(-1,1)$, recalling the construction of Section $2 \mathrm{C}$, we consider the globally hyperbolic spacetime $\widetilde{M}_{s}$, obtained perturbing $M$ with $h_{s}$. Moreover, we take spacelike Cauchy surfaces $\Sigma_{+}, \Sigma_{+}^{\prime}$ for $M_{+}=M \backslash J_{M}^{-}(K)$ and $\Sigma_{-}^{\prime}, \Sigma_{-}$for $M_{-}=M \backslash J_{M}^{+}(K)$ such that $\Sigma_{+}^{\prime} \subseteq I_{M_{+}}^{-}\left(\Sigma_{+}\right)$and $\Sigma_{-}^{\prime} \subseteq I_{M_{-}}^{+}\left(\Sigma_{-}\right)$; see Figure 1. Consider now the diagram in (2-12). For each causal embedding in this diagram, the functor F provides a morphism in PSym, which can be inverted according to the time slice axiom. In particular, we are interested in $\mathscr{F}\left(i_{+}\right)^{-1}: \mathscr{F}(M) \rightarrow \mathscr{F}\left(M_{+}\right)$and $\mathscr{F}\left(j_{-}\right)^{-1}: \mathscr{F}\left(\tilde{M}_{s}\right) \rightarrow \mathscr{F}\left(M_{-}\right)$. These maps can be explicitly defined following the proof of the time slice axiom in Theorem 4.3; see (4-2). For $i_{+}$consider the spacelike Cauchy surfaces $\Sigma_{+}, \Sigma_{+}^{\prime}$ and a partition of unity $\left\{\chi_{+}, \chi_{+}^{\prime}\right\}$ on $M$ such that $\chi_{+}=1$ in $J_{M}^{+}\left(\Sigma_{+}\right)$, while $\chi_{+}^{\prime}=1$ in $J_{M}^{-}\left(\Sigma_{+}^{\prime}\right)$. To define $j_{-}$consider instead $\Sigma_{-}^{\prime}, \Sigma_{-}$, together with a partition of unity $\left\{\chi_{-}^{\prime}, \chi_{-}\right\}$on $\widetilde{M}_{s}$ such that $\chi_{-}^{\prime}=1$ in 
$J_{\widetilde{M}_{s}}^{+}\left(\Sigma_{-}^{\prime}\right)$ and $\chi_{-}=1$ in $J_{\widetilde{M}_{s}}^{-}\left(\Sigma_{-}\right)$. Explicit formulas for $\mathscr{F}\left(j_{+}\right): \mathscr{F}\left(M_{+}\right) \rightarrow \mathscr{F}\left(\tilde{M}_{s}\right)$ and $\mathscr{F}\left(i_{-}\right): \mathscr{F}\left(M_{-}\right) \rightarrow \mathscr{F}(M)$ are obtained simply via pushforward on compactly supported 1-forms as it is explained before Theorem 4.3.

We recall here the explicit form of the maps involved in the definition of the classical relative Cauchy evolution $r_{s}: \mathscr{F}(M) \rightarrow \mathscr{F}(M)$ for the perturbation $h_{s}$ :

$$
\begin{aligned}
\mathscr{F}\left(i_{+}\right)^{-1}: \mathscr{F}(M) \rightarrow \mathscr{F}\left(M_{+}\right), & & {[\alpha] \mapsto\left[i_{+}^{*} \delta \mathrm{d}\left(\chi_{+} G \alpha\right)\right], } \\
\mathscr{F}\left(j_{+}\right): \mathscr{F}\left(M_{+}\right) \rightarrow \mathscr{F}\left(\tilde{M}_{s}\right), & & {[\alpha] \mapsto\left[j_{+*} \alpha\right], } \\
\mathscr{F}\left(j_{-}\right)^{-1}: \mathscr{F}\left(\tilde{M}_{s}\right) \rightarrow \mathscr{F}\left(M_{-}\right), & & {[\alpha] \mapsto\left[-j_{-}^{*} \delta_{s} \mathrm{~d}\left(\chi_{-} G_{s} \alpha\right)\right], } \\
\mathscr{F}\left(i_{-}\right): \mathscr{F}\left(M_{-}\right) \rightarrow \mathscr{F}(M), & & {[\alpha] \mapsto\left[i_{-*} \alpha\right], }
\end{aligned}
$$

where the subscript $s$ means that the perturbation $h_{s}$ plays a role. For example $G_{s}$ is the causal propagator for $\square_{s}=\delta_{s} \mathrm{~d}+\mathrm{d} \delta_{s}$, where $h_{s}$ enters $\delta_{s}$ via the Hodge dual on $\widetilde{M}_{s}$, which is defined out of the perturbed metric $g_{s}=g+h_{s}$ and the orientation of the underlying manifold. Composing the maps above, one gets a formula for the classical relative Cauchy evolution:

$$
r_{s}: \mathscr{F}(M) \rightarrow \mathscr{F}(M), \quad[\alpha] \mapsto\left[-\delta_{s} \mathrm{~d}\left(\chi_{-} G_{s} \delta \mathrm{d}\left(\chi_{+} G \alpha\right)\right)\right]
$$

Remark 5.5. We are dealing with a family $h_{s}$ of perturbations; therefore the above construction should be performed for each $s$. In particular, for each value of $s$, one should consider appropriate spacelike Cauchy surfaces. However, $\operatorname{supp}\left(h_{s}\right) \subseteq K$ for each $s$. Having under control the support of the whole family of perturbations $h_{s}$, it is possible to choose spacelike Cauchy surfaces and partitions of unity which do the job for each $s . \Sigma_{ \pm}, \Sigma_{ \pm}^{\prime}$ were chosen exactly in this spirit.

For the proof of Theorem 5.2 we are interested in the functional derivative of $R_{s}=2\left(r_{s}\right)$. Having this in mind, we compute $\mathrm{d} r_{s}[\alpha] /\left.\mathrm{d} s\right|_{0}$ for an arbitrary, but fixed, $[\alpha] \in \mathscr{F}(M)$. This makes sense at least whenever the topology on $\mathscr{F}(M)$ is Hausdorff; ${ }^{3}$ see Remark 4.5. Equation (5-7) and the Leibniz rule entail that

$$
\left.\frac{\mathrm{d}}{\mathrm{d} s} r_{s}[\alpha]\right|_{0}=\left[-\left.\frac{\mathrm{d}}{\mathrm{d} s} \delta_{s} \mathrm{~d}\left(\chi_{-} G \delta \mathrm{d}\left(\chi_{+} G \alpha\right)\right)\right|_{0}-\left.\frac{\mathrm{d}}{\mathrm{d} s} \delta \mathrm{d}\left(\chi_{-} G_{s} \delta \mathrm{d}\left(\chi_{+} G \alpha\right)\right)\right|_{0}\right] .
$$

Since $\operatorname{supp}\left(h_{s}\right) \cap \operatorname{supp}\left(\chi_{-}\right) \subseteq K \cap M_{-}=\varnothing$, the argument of the first derivative is constant in $s$. We deduce that the first contribution vanishes. Decomposing $G_{s}$ in $G_{s+}-G_{s-}$ and noting that $\chi_{-} G_{s+} \delta \mathrm{d}\left(\chi_{+} G \alpha\right)$ and $\chi_{-}^{\prime} G_{s-} \delta \mathrm{d}\left(\chi_{+} G \alpha\right)$ have compact supports, we get

${ }^{3}$ This property ensures uniqueness of limits; therefore $\mathrm{d} r_{s}[\alpha] /\left.\mathrm{d} s\right|_{0}$ is uniquely defined as the limit for $s \rightarrow 0$ of $\left(r_{s}[\alpha]-[\alpha]\right) / s$. 


$$
\begin{aligned}
\left.\frac{\mathrm{d}}{\mathrm{d} s} r_{s}[\alpha]\right|_{0} & =\left[-\left.\frac{\mathrm{d}}{\mathrm{d} s} \delta \mathrm{d}\left(\chi_{-} G_{s+} \delta \mathrm{d}\left(\chi_{+} G \alpha\right)\right)\right|_{0}+\left.\frac{\mathrm{d}}{\mathrm{d} s} \delta \mathrm{d}\left(\chi_{-} G_{s} \delta \mathrm{d}\left(\chi_{+} G \alpha\right)\right)\right|_{0}\right] \\
& =\left[\left.\frac{\mathrm{d}}{\mathrm{d} s} \delta \mathrm{d} G_{s-} \delta \mathrm{d}\left(\chi_{+} G \alpha\right)\right|_{0}\right]=\left[-\left.\frac{\mathrm{d}}{\mathrm{d} s} \delta \mathrm{d} G_{s-} \square\left(\chi_{+}^{\prime} G \alpha\right)\right|_{0}\right]
\end{aligned}
$$

due to the fact that $\delta \mathrm{d} \Omega_{\mathrm{c}}^{1}(M)$ is identified with 0 in $\mathscr{F}(M), \mathrm{d} G_{s-}=G_{s_{-}} \mathrm{d}$ on $\Omega_{\mathrm{c}}^{k}(M)$ and $\square\left(\chi_{+} G \alpha\right)=-\square\left(\chi_{+}^{\prime} G \alpha\right)$. On account of the properties of the Green operators (2-6), and exploiting the Leibniz rule, one obtains the identity

$$
\left.\frac{\mathrm{d}}{\mathrm{d} s} G_{s-} \square\left(\chi_{+}^{\prime} G \alpha\right)\right|_{0}+\left.G_{-} \frac{\mathrm{d}}{\mathrm{d} s} \square_{s}\left(\chi_{+}^{\prime} G \alpha\right)\right|_{0}=\left.\frac{\mathrm{d}}{\mathrm{d} s} G_{s-} \square_{s}\left(\chi_{+}^{\prime} G \alpha\right)\right|_{0}=0,
$$

which can be plugged into (5-8). Keeping in mind that $\operatorname{supp}\left(h_{s}\right)$ does not meet $\operatorname{supp}\left(\chi_{+}\right)$, one concludes that $\square_{s}\left(\chi_{+} G \alpha\right)=\square\left(\chi_{+} G \alpha\right)$ for each $s$. Thus, taking into account also that $\square\left(\chi_{+} G \alpha\right)=-\square\left(\chi_{-} G \alpha\right)$ has compact support, we get the equality

$$
\left.\frac{\mathrm{d}}{\mathrm{d} s} r_{s}[\alpha]\right|_{0}=\left[\left.\delta \mathrm{d} G_{-} \frac{\mathrm{d}}{\mathrm{d} s} \square_{s}\left(\chi_{+}^{\prime} G \alpha\right)\right|_{0}\right]=\left[\left.\delta \mathrm{d} G_{-} \frac{\mathrm{d}}{\mathrm{d} s} \delta_{s} \mathrm{~d} G \alpha\right|_{0}\right] .
$$

From $\delta_{s} \delta_{s}=0$, the Leibniz rule and $\delta \alpha=0$ we deduce that

$$
\left.\delta \frac{\mathrm{d}}{\mathrm{d} s} \delta_{s} \mathrm{~d} G \alpha\right|_{0}=\left.\frac{\mathrm{d}}{\mathrm{d} s} \delta \delta_{s} \mathrm{~d} G \alpha\right|_{0}+\left.\frac{\mathrm{d}}{\mathrm{d} s} \delta_{s} \delta \mathrm{d} G \alpha\right|_{0}=\left.\frac{\mathrm{d}}{\mathrm{d} s} \delta_{s} \delta_{s} \mathrm{~d} G \alpha\right|_{0}=0 .
$$

Taking into account this information, from (5-9) we come to the conclusion:

$$
\left.\frac{\mathrm{d}}{\mathrm{d} s} r_{s}[\alpha]\right|_{0}=\left[\left.\square G_{-} \frac{\mathrm{d}}{\mathrm{d} s} \delta_{s} \mathrm{~d} G \alpha\right|_{0}\right]=\left[\left.\frac{\mathrm{d}}{\mathrm{d} s} \delta_{s} \mathrm{~d} G \alpha\right|_{0}\right] .
$$

Explicitly, introducing $F=\mathrm{d} G \alpha$, one reads

$$
\begin{aligned}
\left(\left.\frac{\mathrm{d}}{\mathrm{d} s} \delta_{s} F\right|_{0}\right)_{\rho} & =-\left.\frac{\mathrm{d}}{\mathrm{d} s} g_{s}^{\mu \nu} \nabla_{s \mu} F_{v \rho}\right|_{0} \\
& =\dot{h}_{\mu \nu} \nabla^{\mu} F_{\rho}^{\nu}+\frac{1}{2}\left(F_{\rho}^{\mu} \nabla^{v} \dot{h}_{\mu \nu}-F_{\nu}^{\mu} \nabla^{v} \dot{h}_{\mu \rho}\right),
\end{aligned}
$$

where $\nabla$ and $\nabla_{s}$ are the Levi-Civita connections respectively for the unperturbed metric $g$ and the perturbed one $g_{s}$. All indices in the result are raised using $g$ and $\dot{h}$ denotes $\mathrm{d} h_{s} /\left.\mathrm{d} s\right|_{0}$. This result follows from the subsequent identities, which are trivial consequences of $g_{s}^{\mu \nu}$ being the inverse of $g_{s \mu \nu}$ and $\nabla\left(\nabla_{s}\right)$ being the Levi-Civita connection for $g$ (respectively $g_{s}$ ):

$$
\begin{aligned}
\left.\frac{\mathrm{d}}{\mathrm{d} s} g_{s}^{\mu \nu}\right|_{0} & =-g^{\mu \rho} g^{\nu \sigma} \dot{h}_{\rho \sigma}, \\
\left.\frac{\mathrm{d}}{\mathrm{d} s}\left(\nabla_{s \mu} X^{\rho}-\nabla_{\mu} X^{\rho}\right)\right|_{0} & =\frac{1}{2} X^{v} g^{\rho \sigma} \nabla_{\mu} \dot{h}_{\nu \sigma},
\end{aligned}
$$

for each vector field $X$ on $M$. 
5C. Proof of Theorem 5.2. According to the hypotheses, let us consider a globally hyperbolic spacetime $M$ and a quasifree Hadamard state $\omega$ on $\mathscr{A}(M)$ with associated GNS triple $\left(D_{\omega}, \pi_{\omega}, \Omega_{\omega}\right)$. Fixing $[\alpha] \in \mathscr{F}(M), \theta \in D_{\omega}, K$ compact in $M$, a 1-parameter family $s \in(-1,1) \mapsto h_{s} \in \mathrm{hp}(M)$ supported inside $K$ and taking into account Section 5A and Section 5B, the claim of the theorem boils down to the identity below:

$$
\left\langle\theta, \phi_{\omega}\left(\left.\frac{\mathrm{d}}{\mathrm{d} s} r_{s}[\alpha]\right|_{0}\right) \theta\right\rangle=\frac{\mathrm{i}}{2} \int_{M}\left\langle\theta,\left[\hat{T}^{\mu \nu}, \phi_{\omega}([\alpha])\right] \theta\right\rangle \dot{h}_{\mu \nu} \text { vol, }
$$

where $\dot{h}$ stands for $\mathrm{d} h_{s} /\left.\mathrm{d} s\right|_{0}$. We rewrite the left side using (5-4) for $n=1$, together with eqs. (5-10) and (5-11), and introducing the notation $A=G \alpha, F=\mathrm{d} A$ and $\tilde{A}=\langle\theta, \hat{A} \theta\rangle$. For the right side we consider (5-6), keeping in mind that $\dot{h}_{\mu \nu}=\dot{h}_{\nu \mu}$ and defining $\tilde{F}=\mathrm{d} \tilde{A}$. This turns (5-14) into the following identity:

$$
\begin{aligned}
& L \doteq \int_{M} \tilde{A}^{\rho} {\left[\dot{h}_{\mu \nu} \nabla^{\mu} F_{\rho}^{v}{ }_{\rho}^{\frac{1}{2}}\left(F_{\rho}^{\mu} \nabla^{v} \dot{h}_{\mu \nu}-F^{\mu}{ }_{\nu} \nabla^{v} \dot{h}_{\mu \rho}\right)\right] \mathrm{vol} } \\
&=-\frac{1}{2} \int_{M} \dot{h}_{\mu \nu}\left(2 F^{\mu \rho} \tilde{F}_{\rho}^{\nu}-\frac{1}{2} g^{\mu \nu} F^{\rho \sigma} \tilde{F}_{\rho \sigma}\right) \mathrm{vol} \doteq R .
\end{aligned}
$$

The proof will be complete as soon as one manages to check this identity.

We start by considering the right side. Here we integrate by parts all covariant derivatives acting on $\tilde{A}$. Note that several terms arising from partial integration vanish on account of $\delta F=\delta \mathrm{d} G \alpha=0$. On account of the symmetry of $\dot{h}$ and the antisymmetry of $F$, the result is

$$
\begin{aligned}
R=\int_{M} \dot{h}_{\mu \nu} \tilde{A}_{\rho} \nabla^{v} F^{\mu \rho} \mathrm{vol}+\int_{M} F^{\mu \rho}\left(\tilde{A}_{\rho} \nabla^{v} \dot{h}_{\mu \nu}-\tilde{A}^{v} \nabla_{\rho} \dot{h}_{\mu \nu}\right) \text { vol } & \\
& +\frac{1}{2} \int_{M} g^{\mu v} F^{\rho \sigma} \tilde{A}_{\rho} \nabla_{\sigma} \dot{h}_{\mu \nu} \text { vol. }
\end{aligned}
$$

Comparing $R$ with the left side of (5-15), one reads

$$
\begin{aligned}
R & =L+\frac{1}{2} \int_{M} F^{\mu \rho}\left(\tilde{A}_{\rho} \nabla^{v} \dot{h}_{\mu \nu}-\tilde{A}^{v} \nabla_{\rho} \dot{h}_{\mu \nu}\right) \text { vol }+\frac{1}{2} \int_{M} g^{\mu \nu} F^{\rho \sigma} \tilde{A}_{\rho} \nabla_{\sigma} \dot{h}_{\mu \nu} \text { vol } \\
& =L+\frac{1}{2} \int_{M} F^{\rho \sigma} \tilde{A}_{\rho}\left(g^{\mu \nu} \nabla_{\sigma} \dot{h}_{\mu \nu}-\nabla^{v} \dot{h}_{\sigma \nu}\right) \text { vol }-\frac{1}{2} \int_{M} \tilde{A}^{v} F^{\mu \rho} \nabla_{\rho} \dot{h}_{\mu \nu} \text { vol } \\
& \doteq L+\frac{1}{2} \int_{M} F^{\rho \sigma} \tilde{A}_{\rho} X_{\sigma} \text { vol }-\frac{1}{2} \int_{M} \tilde{A}^{v} Y_{\nu} \text { vol },
\end{aligned}
$$

$X$ and $Y$ being defined by

$$
X_{\sigma}=g^{\mu \nu} \nabla_{\sigma} \dot{h}_{\mu \nu}-\nabla^{v} \dot{h}_{\sigma v}, \quad Y_{v}=F^{\mu \rho} \nabla_{\rho} \dot{h}_{\mu \nu} .
$$

The rest of the proof is devoted to showing that both $X$ and $Y$ vanish everywhere on $M$. (5-13) entails that 


$$
\left.\frac{\mathrm{d}}{\mathrm{d} s} \Gamma_{s \mu \nu}^{\rho}\right|_{0}=\frac{1}{2} g^{\rho \sigma} \nabla_{\mu} \dot{h}_{\nu \sigma},
$$

$\Gamma_{s}$ being the Christoffel symbols for the connection $\nabla_{s}$. As a consequence, $\nabla_{\mu} \dot{h}_{\nu \sigma}$ is symmetric upon the interchange of $\mu$ and $\nu$. Taking into account that $F$ is antisymmetric, we get $X=0$ and $Y=0$, thus concluding the proof.

\section{Acknowledgements}

The author wishes to thank his supervisor Claudio Dappiaggi for the enlightening discussions and suggestions. This research was supported by a Ph.D. scholarship from the University of Pavia.

\section{References}

[Bär 2013] C. Bär, “Green-hyperbolic operators on globally hyperbolic spacetimes”, preprint, 2013. arXiv 1310.0738

[Bär and Becker 2009] C. Bär and C. Becker, " $C^{*}$-algebras", pp. 1-37 in Quantum field theory on curved spacetimes, Lecture Notes in Phys. 786, Springer, Berlin, 2009.

[Bär and Ginoux 2012a] C. Bär and N. Ginoux, "Classical and quantum fields on Lorentzian manifolds”, pp. 359-400 in Global differential geometry, edited by C. Bär et al., Springer Proc. in Math. 17, Springer, Berlin, 2012.

[Bär and Ginoux 2012b] C. Bär and N. Ginoux, "CCR- versus CAR-quantization on curved spacetimes", pp. 183-206 in Quantum field theory and gravity, edited by F. Finster et al., Birkhäuser, Basel, 2012.

[Bär et al. 2007] C. Bär, N. Ginoux, and F. Pfäffle, Wave equations on Lorentzian manifolds and quantization, European Mathematical Society (EMS), Zürich, 2007.

[Beem et al. 1996] J. K. Beem, P. E. Ehrlich, and K. L. Easley, Global Lorentzian geometry, 2nd ed., Pure Appl. Math. 202, Marcel Dekker, New York, 1996.

[Benini 2014] M. Benini, "Optimal space of linear classical observables for Maxwell $k$-forms via spacelike and timelike compact de Rham cohomologies", preprint, 2014. arXiv 1401.7563

[Benini et al. 2013] M. Benini, C. Dappiaggi, and T.-P. Hack, "Quantum field theory on curved backgrounds-a primer”, Internat. J. Modern Phys. A 28:17 (2013), 1330023, 49.

[Benini et al. 2014a] M. Benini, C. Dappiaggi, T.-P. Hack, and A. Schenkel, "A C*-Algebra for Quantized Principal U(1)-Connections on Globally Hyperbolic Lorentzian Manifolds", Communications in Mathematical Physics (2014), 1-28.

[Benini et al. 2014b] M. Benini, C. Dappiaggi, and A. Schenkel, "Quantized abelian principal connections on Lorentzian manifolds”, Comm. Math. Phys. 330:1 (2014), 123-152.

[Benini et al. 2014c] M. Benini, C. Dappiaggi, and A. Schenkel, "Quantum field theory on affine bundles", Ann. Henri Poincaré 15:1 (2014), 171-211.

[Bernal and Sánchez 2005] A. N. Bernal and M. Sánchez, "Smoothness of time functions and the metric splitting of globally hyperbolic spacetimes”, Comm. Math. Phys. 257:1 (2005), 43-50.

[Borchers 1962] H.-J. Borchers, "On structure of the algebra of field operators", Nuovo Cimento (10) 24 (1962), 214-236.

[Bott and Tu 1982] R. Bott and L. W. Tu, Differential forms in algebraic topology, Graduate Texts in Mathematics 82, Springer, New York, 1982. 
[Bratteli and Robinson 1987] O. Bratteli and D. W. Robinson, Operator algebras and quantum statistical mechanics, 1 : $C^{*}$ - and $W^{*}$-algebras, symmetry groups, decomposition of states, 2 nd ed., Springer, New York, 1987.

[Brunetti et al. 2003] R. Brunetti, K. Fredenhagen, and R. Verch, "The generally covariant locality principle_a new paradigm for local quantum field theory", Comm. Math. Phys. 237:1-2 (2003), $31-68$.

[Brunetti et al. 2012] R. Brunetti, K. Fredenhagen, and P. L. Ribeiro, "Algebraic structure of classical field theory, I: Kinematics and linearized dynamics for real scalar fields", preprint, 2012. arXiv 1209.2148

[Dappiaggi 2011] C. Dappiaggi, "Remarks on the Reeh-Schlieder property for higher spin free fields on curved spacetimes”, Rev. Math. Phys. 23:10 (2011), 1035-1062.

[Dappiaggi and Lang 2012] C. Dappiaggi and B. Lang, "Quantization of Maxwell's equations on curved backgrounds and general local covariance", Lett. Math. Phys. 101:3 (2012), 265-287.

[Dappiaggi and Siemssen 2013] C. Dappiaggi and D. Siemssen, "Hadamard states for the vector potential on asymptotically flat spacetimes", Rev. Math. Phys. 25:1 (2013), 1350002, 31.

[Dimock 1980] J. Dimock, "Algebras of local observables on a manifold", Comm. Math. Phys. 77:3 (1980), 219-228.

[Dimock 1992] J. Dimock, "Quantized electromagnetic field on a manifold", Rev. Math. Phys. 4:2 (1992), 223-233.

[Fewster and Lang 2014] C. J. Fewster and B. Lang, "Dynamical locality of the free Maxwell field", preprint, 2014. arXiv 1403.7083

[Fewster and Pfenning 2003] C. J. Fewster and M. J. Pfenning, "A quantum weak energy inequality for spin-one fields in curved space-time", J. Math. Phys. 44:10 (2003), 4480-4513.

[Fewster and Verch 2012] C. J. Fewster and R. Verch, "Dynamical locality and covariance: what makes a physical theory the same in all spacetimes?", Ann. Henri Poincaré 13:7 (2012), 16131674.

[Finster and Strohmaier 2013] F. Finster and A. Strohmaier, "Gupta-Bleuler quantization of the Maxwell field in globally hyperbolic space-times”, preprint, 2013. arXiv 1307.1632

[Fulling et al. 1978] S. A. Fulling, M. Sweeny, and R. M. Wald, "Singularity structure of the twopoint function quantum field theory in curved spacetime", Comm. Math. Phys. 63:3 (1978), 257264.

[Fulling et al. 1981] S. A. Fulling, F. J. Narcowich, and R. M. Wald, "Singularity structure of the two-point function in quantum field theory in curved spacetime, II", Ann. Physics 136:2 (1981), 243-272.

[Haag and Kastler 1964] R. Haag and D. Kastler, "An algebraic approach to quantum field theory", J. Mathematical Phys. 5 (1964), 848-861.

[Hack and Schenkel 2013] T.-P. Hack and A. Schenkel, "Linear bosonic and fermionic quantum gauge theories on curved spacetimes", Gen. Relativity Gravitation 45:5 (2013), 877-910.

[Hörmander 2003] L. Hörmander, The analysis of linear partial differential operators, I: Distribution theory and Fourier analysis, 2nd ed., Springer, Berlin, 2003.

[Khavkine 2014a] I. Khavkine, "Cohomology with causally restricted supports", preprint, 2014. arXiv 1404.1932

[Khavkine 2014b] I. Khavkine, "Covariant phase space, constraints, gauge and the Peierls formula", Internat. J. Modern Phys. A 29:5 (2014), \#1430009. 
[MacLane 1971] S. MacLane, Categories for the working mathematician, Graduate Texts in Mathematics 5, Springer, New York, 1971.

[Manuceau et al. 1973] J. Manuceau, M. Sirugue, D. Testard, and A. Verbeure, "The smallest $C^{*}$ algebra for canonical commutations relations”, Comm. Math. Phys. 32 (1973), 231-243.

[Pfenning 2009] M. J. Pfenning, "Quantization of the Maxwell field in curved spacetimes of arbitrary dimension”, Classical Quantum Gravity 26:13 (2009), 135017, 20.

[Radzikowski 1996a] M. J. Radzikowski, "Micro-local approach to the Hadamard condition in quantum field theory on curved space-time”, Comm. Math. Phys. 179:3 (1996), 529-553.

[Radzikowski 1996b] M. J. Radzikowski, "A local-to-global singularity theorem for quantum field theory on curved space-time", Comm. Math. Phys. 180:1 (1996), 1-22.

[Sanders 2010] K. Sanders, "The locally covariant Dirac field”, Rev. Math. Phys. 22:4 (2010), 381430.

[Sanders 2013] K. Sanders, "A note on spacelike and timelike compactness", Classical Quantum Gravity 30:11 (2013), 115014, 10.

[Sanders et al. 2014] K. Sanders, C. Dappiaggi, and T.-P. Hack, "Electromagnetism, local covariance, the Aharonov-Bohm effect and Gauss' law", Comm. Math. Phys. 328:2 (2014), 625-667.

[Uhlmann 1962] A. Uhlmann, "Über die Definition der Quantenfelder nach Wightman und Haag", Wiss. Z. Karl-Marx-Univ. Leipzig Math.-Nat. Reihe 11 (1962), 213-217.

[Wald 1994] R. M. Wald, Quantum field theory in curved spacetime and black hole thermodynamics, University of Chicago Press, 1994.

[Waldmann 2012] S. Waldmann, "Geometric wave equations”, preprint, 2012. arXiv 1208.4706

Received 17 Feb 2014. Accepted 21 Apr 2014.

MARCO BENINI: marco.benini@pv.infn.it

Dipartimento di Fisica, Università di Pavia \& INFN, Sezione di Pavia, Via Bassi 6, I-27100 Pavia, Italy

marco.benini@pv.infn.it 


\section{EDITORIAL BOARD}

ANTONIO CARCATERRA ERIC A. CARLEN

FRANCESCO DELL'ISOLA

RAFFAELE ESPOSITO

ALBERT FANNJIANG

Gilles A. FrancFort

PiERANGElo MARCATI

JEAN-JACQUES MARIGO

PETER A. MARKOWICH MARTIN OSTOJA-STARZEWSKI

PIERRE SEPPECHER

DAVID J. STEIGMANN

PAUl STEINMANN

PierRe M. Suquet

\section{MANAGING EDITORS}

MICOL AMAR

CORRADO LATTANZIO

ANGELA MADEO

MARTIN OSTOJA-STARZEWSKI

\section{ADVISORY BOARD}

ADNAN AKAY

Holm AltenbaCH

MICOL AMAR

HARM ASKES

TEODOR ATANACKOVIĆ

VICTOR BERDICHEVSKY

GuY BouchitTÉ

ANDREA BRAIDES

ROBERTO CAMASSA

MAURO CARFORE

ERIC DARVE

FELIX DARVE

ANNA DE MASI

Gianpietro Del Piero

EMMANUELE Di BENEDETTO

BERNOLD FIEDLER

IRENE M. GAMBA

SERGEY GAVRILYUK

TIMOTHY J. HEALEY

DOMINIQUE JEULIN

ROGER E. KHAYAT

CORRADO LATTANZIO

ROBERT P. LIPTON

ANGELO LUONGO

ANGEla MadeO

JUAN J. MANFREDI

CARLO MARCHIORO

GÉrard A. MAUGin

ROBERTO NATALINI

PATRIZIO NEFF

ANDREY PIATNITSKI

ERrico Presutti

MARIO PUlVIRENTI

LuCiO RuSSO

Miguel A. F. SANJUAN

Patrick SElvadurai

ALEXANDER P. SEYRANIAN

MIROSLAV ŠILHAVÝ

GUIDO SWEERS

ANTOINETTE TORDESILLAS

LEV TRUSKINOVSKY

JUAN J. L. VELÁZQUEZ

VINCENZO VESPRI

ANGELO VULPIANI msp.org/memocs

Università di Roma “La Sapienza”, Italia

Rutgers University, USA

(CO-CHAIR) Università di Roma "La Sapienza", Italia

(TREASURER) Università dell'Aquila, Italia

University of California at Davis, USA

(CO-CHAIR) Université Paris-Nord, France

Università dell' Aquila, Italy

École Polytechnique, France

DAMTP Cambridge, UK, and University of Vienna, Austria

(CHAIR MANAGING EDITOR) Univ. of Illinois at Urbana-Champaign, USA

Université du Sud Toulon-Var, France

University of California at Berkeley, USA

Universität Erlangen-Nürnberg, Germany

LMA CNRS Marseille, France

Università di Roma “La Sapienza”, Italia

Università dell' Aquila, Italy

Université de Lyon-INSA (Institut National des Sciences Appliquées), France (CHAIR MANAGING EDITOR) Univ. of Illinois at Urbana-Champaign, USA

Carnegie Mellon University, USA, and Bilkent University, Turkey

Otto-von-Guericke-Universität Magdeburg, Germany

Università di Roma "La Sapienza”, Italia

University of Sheffield, UK

University of Novi Sad, Serbia

Wayne State University, USA

Université du Sud Toulon-Var, France

Università di Roma Tor Vergata, Italia

University of North Carolina at Chapel Hill, USA

Università di Pavia, Italia

Stanford University, USA

Institut Polytechnique de Grenoble, France

Università dell'Aquila, Italia

Università di Ferrara and International Research Center MEMOCS, Italia

Vanderbilt University, USA

Freie Universität Berlin, Germany

University of Texas at Austin, USA

Université Aix-Marseille, France

Cornell University, USA

École des Mines, France

University of Western Ontario, Canada

Università dell' Aquila, Italy

Louisiana State University, USA

Università dell' Aquila, Italia

Université de Lyon-INSA (Institut National des Sciences Appliquées), France

University of Pittsburgh, USA

Università di Roma "La Sapienza", Italia

Université Paris VI, France

Istituto per le Applicazioni del Calcolo "M. Picone", Italy

Universität Duisburg-Essen, Germany

Narvik University College, Norway, Russia

Università di Roma Tor Vergata, Italy

Università di Roma "La Sapienza", Italia

Università di Roma “Tor Vergata", Italia

Universidad Rey Juan Carlos, Madrid, Spain

McGill University, Canada

Moscow State Lomonosov University, Russia

Academy of Sciences of the Czech Republic

Universität zu Köln, Germany

University of Melbourne, Australia

École Polytechnique, France

Bonn University, Germany

Università di Firenze, Italia

Università di Roma La Sapienza, Italia

MEMOCS (ISSN 2325-3444 electronic, 2326-7186 printed) is a journal of the International Research Center for the Mathematics and Mechanics of Complex Systems at the Università dell'Aquila, Italy.

Cover image: "Tangle" by @ John Horigan; produced using the Context Free program (contextfreeart.org).

PUBLISHED BY

mathematical sciences publishers

nonprofit scientific publishing

http://msp.org/

(C) 2015 Mathematical Sciences Publishers 
Mathematics and Mechanics of Complex Systems vol. 3 no. 2

Derivation of nonlinear shell models combining shear and 101 flexure: application to biological membranes

Olivier Pantz and Karim Trabelsi

Canonical duality theory and triality for solving general global optimization problems in complex systems

Daniel Morales-Silva and David Y. Gao

Neutrality of eccentrically coated elastic inclusions

$\mathrm{Xu}$ Wang and Peter Schiavone

Relative Cauchy evolution for the vector potential on globally hyperbolic spacetimes

Marco Benini

MEMOCS is a journal of the International Research Center for the Mathematics and Mechanics of Complex Systems at the Università dell' Aquila, Italy.

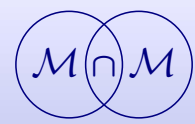

